\title{
Long-term photometric monitoring with the Mercator telescope
}

\section{Frequencies and multicolour amplitudes of $\gamma$ Doradus stars $\star, \star \star$}

\author{
J. Cuypers ${ }^{1}$, C. Aerts ${ }^{2,3}$, P. De Cat ${ }^{1}$, J. De Ridder ${ }^{2}$, K. Goossens ${ }^{2}$, C. Schoenaers ${ }^{1,4}$, K. Uytterhoeven ${ }^{2,5,9}$, B. Acke ${ }^{2, \star \star \star}$, \\ G. Davignon ${ }^{2,5}$, J. Debosscher ${ }^{2}$, L. Decin ${ }^{2, * * *}$, W. De Meester ${ }^{2}$, P. Deroo ${ }^{2,10, \dagger}$, R. Drummond ${ }^{2,8}$, K. Kolenberg ${ }^{2,7}$, \\ K. Lefever ${ }^{2,8}$, G. Raskin ${ }^{2,5}$, M. Reyniers ${ }^{2,6}$, S. Saesen ${ }^{2, * * *}$, B. Vandenbussche ${ }^{2}$, R. Van Malderen ${ }^{2,6}$, T. Verhoelst ${ }^{2, * * *}$, \\ H. Van Winckel ${ }^{2}$, and C. Waelkens ${ }^{2}$
}

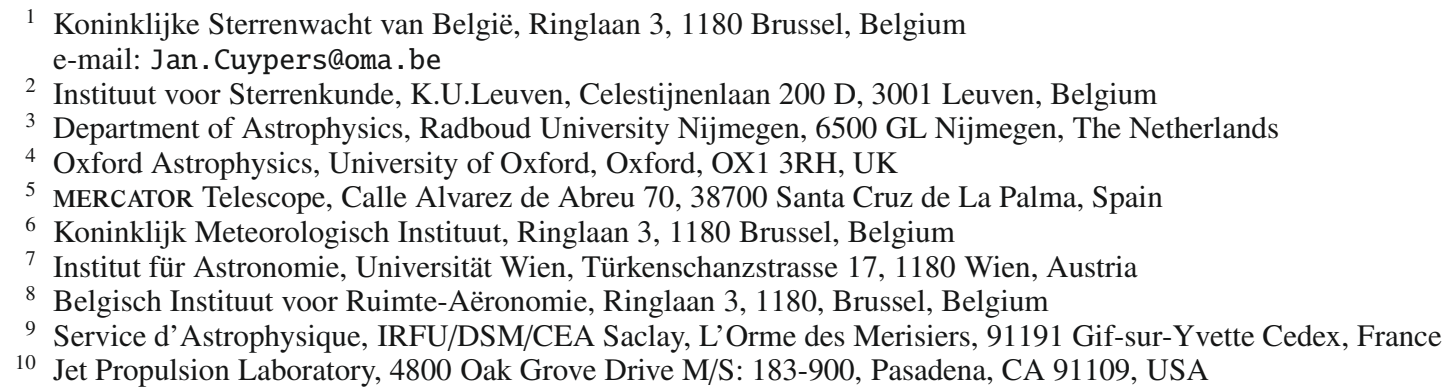

Received 20 January 2009 / Accepted 5 March 2009

\section{ABSTRACT}

\begin{abstract}
Context. $\gamma$ Dor stars are excellent targets for asteroseismology since the gravity modes present in these stars probe the deep stellar interiors. Mode identification will improve the knowledge of these stars considerably.

Aims. A selected group of $\gamma$ Dor stars and some candidates were observed with the Mercator telescope to find and/or confirm the periodicities in the light variations and to derive reliable amplitude ratios in different pass bands.

Methods. A frequency analysis was performed on all new data obtained in the Geneva photometric system. In order to have more reliable and accurate frequencies, the new data were combined with similar data from the literature and with Hipparcos observations. A set of frequencies that minimized the residuals in a harmonic fit was searched for while allowing means and amplitudes to vary from one observation set to another.

Results. Frequencies and amplitudes in the photometric passbands of the Geneva system are given for $21 \gamma$ Dor stars. We report the discovery of HD 74504 as a newly found $\gamma$ Dor star.

Conclusions. Our study provides the first extensive multicolour database for the understanding of gravity modes in F-type stars.
\end{abstract}

Key words. stars: variables: general - stars: oscillations - stars: individual: $\gamma$ Doradus - techniques: photometric

\section{Introduction: $\gamma$ Doradus stars ( $\gamma$ Dor stars)}

As reviewed in the article by Henry et al. (2007) the class of $\gamma$ Dor stars has now over 60 members. These stars have spectral types of late A or F, luminosity class IV or V and exhibit periodicity in the light variations with periods in the range of 0.3 to 3 days. Line-profile variability is present as well (Mathias et al. 2004; De Cat et al. 2006). There is no doubt that the cause of the variations is pulsation. The modes are high-order gravity modes (g-modes), excited by a flux blocking mechanism at the base of

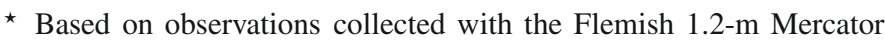
Telescope at Roque de los Muchachos, La Palma.

$\star \star$ Tables 5 to 25 are also available at the CDS via anonymous ftp to cdsarc.u-strasbg.fr $(130.79 .128 .5)$ or via http://cdsweb.u-strasbg. fr/cgi-bin/qcat?]/A+A/499/967

$\star \star \star$ Fellow of the Fund for Scientific Research, Flanders (FWO).

NASA Post-doctoral fellow.
}

the convective envelope of the stars (Guzik et al. 2000; Dupret et al. 2005).

Because the g-modes probe the deep stellar interior, the $\gamma$ Dor stars are excellent targets for asteroseismology, particularly since they may exhibit solar-like oscillations as well (Michel et al. 2008). However, mode identificiation is not simple for these stars since the spectrum of g-modes is very dense and only a few modes seem to have high enough amplitudes to be well observed from the ground. Therefore, observables such as photometric amplitude ratios and line-profile variations are extremely useful to identify the modes. By observing light variations in different passbands the identification of the degree $\ell$ of the pulsation mode becomes possible (Balona \& Stobie 1979; Watson 1988; Dupret et al. 2005).

Since this kind of observable is made available with this paper for $21 \gamma$ Dor stars, a large number of variable stars of this class will have their modes identified, and parameters for the 
physics of stellar models, including e.g. a value for the mixinglength parameter, can in principle be derived. Such seismic inferences are the subject of an accompanying paper (Miglio et al. 2009 , in preparation).

\section{The instrument and the observations}

The Mercator telescope is a 1.2-m telescope located on the Roque de los Muchachos observatory on La Palma, Spain. Since the beginning of its scientific operations in spring 2001 it was equipped with the P7 photometer.

The P7 photometer is the refurbished photometer that has been active on the $70-\mathrm{cm}$ Swiss Telescope at La Silla Chile before. It is a two-channel photometer for quasi simultaneous 7-band measurements $\left(U, B_{1}, B, B_{2}, V_{1}, V, G\right)$ in the Geneva photometric system (Golay 1966; Rufener \& Nicolet 1988; Rufener 1989). The first channel (A) is centred on the star while the second channel (B) is centred on the sky. The filter wheel turns at 4 hertz and a chopper directs both channels alternatively to the photomultiplier. As such, the photomultiplier measures both beams through the seven filters four times per second. In this way quasi-simultaneous measurements of the light in the 7 filters become possible. This makes the instrument very suitable to observe variable stars, since information on the light variations in all filters can be obtained very efficiently.

Furthermore an almost continuous access to the telescope during the year was possible, so the instrument became ideal to monitor stars with periods of the order of days and/or long beat periods of the order of months or years. Here we report on the results of a long-term monitoring of $\gamma$ Dor stars, while De Cat et al. (2007) discussed the results for a sample of variable OB stars.

Some of these stars were observed over two years (time span around 370 days), some over three years (time span around 740 days) or four years (1110 days). Stars observed during only one season were not considered in detail. Up to five observations per star were obtained for about two months per season. This resulted in about 130 measurements per star on average, but there is a large spread as can be seen in Table 1 .

Photometric observations in the Geneva system are labeled with two quality indices (from lowest quality 0 to highest quality 4), one for the magnitudes, one for the colours (Rufener 1989). In a first analysis we eliminated all observations labeled 0 or 1 , but we also carried out a re-analysis with the low-quality observations included. In most cases the difference in the result was negligible. Since some stars were only measured at high airmasses, they almost all automatically received a low index value. Here we used those values as well, since without them the number of observations of those stars would be too low. The errors are slightly larger in such cases, but the results are the same. Observations of standard stars indicate that the errors for the programme stars, which have visual magnitudes between 7 and 9 , are in the range 0.005 to $0.010 \mathrm{mag}$.

\section{Selection of the targets}

A selection of stars was made out of the lists by Aerts et al. (1998) and Handler (1999), of the Hipparcos selection results and from lists of $\gamma$ Dor stars, candidate $\gamma$ Dor or other variables (Koen \& Eyer 2002). Since the start of the observations, almost all stars of the list were comfirmed as $\gamma$ Dor stars. For very few of them, multicolour photometry was available and in several cases the multiperiodicity needed confirmation. The list with the 21 stars with more than 25 observations of good quality, spread over more than one season, is given in Table 1. A number of stars was already (partly) analysed in a previous paper (De Ridder et al. 2004) but for some stars the additional observations proved to be very useful to clarify the multiperiodicity of the light variations.

Fifteen other variable A-F stars were monitored during the same period. Not all showed characteristics of $\gamma$ Dor stars: a few turned out to be $\delta$ Scuti stars, others need some further analysis. The results for these stars will be published in another article.

\section{Analysis methods}

We used standard methods for the period analysis, but since the light curves of these stars are very sinusoidal, we adopted as a final analysis method a multifrequency least-squares fit with sinusoidal components (Schoenaers \& Cuypers 2004), where we searched for the best frequencies in large intervals around an initial solution. This is an extremely computer intensive procedure since the sum of the residuals is a rapidly oscillating function, especially when up to six frequencies had to be considered. To obtain the initial values we used Lomb-Scargle periodogram methods (Lomb 1976; Scargle 1982), and phase dispersion minimization methods (Stellingwerf 1978), each time followed by a prewhitening for a first analysis. The final search was always done with a multifrequency least-squares fit.

For initial values of the frequencies in the Mercator data we usually looked at the B or B1 data, since we expect the highest amplitudes of the light variations in these filters (e.g. Aerts et al. 2004). It happened that the amplitude in other filters (typically $\mathrm{V} 1$ or $\mathrm{G}$ ) became too low for the frequencies to be significant there. We re-analysed the Hipparcos data independently, but we looked only for a local minimum in the least-squares fit in the (wide) neighbourhood of the ground-based solution.

If observations were available from other sources, we combined the Geneva colour $V$, Johnson $V$ and Hipparcos data. We searched in the combined data for a multifrequency least-squares fit with unknown means and amplitudes for each data source to search for the best frequency (frequencies) to describe all data. We did not use statistical weights on individual observations or groups of data: the data from the literature were of similar quality and the measurement errors about the same as for the Geneva photometry. The Hipparcos data often had larger errors, but since these are important for solving the alias problems, we also gave those equal weights.

Although for individual data sets only frequencies peaking four times above the average signal-to-noise level in the periodogram are acceptable, we relaxed this criterion for the frequencies found with the multifrequency fit technique in the combined data. Frequency solutions of the multifrequency fit were accepted, if a corresponding extremum was found in the periodogram of each data set. In this way we accepted frequencies with peaks 1.5 times above the noise in some of the individual data sets, as shown a posteriori.

In several cases the combination of the data solved the alias or ambiguity problems and it gave, in general, more accurate values for the frequencies. In a few cases, it turned out that observations of the same star(s) were carried out, unintentionally, as in multisite campaigns. Here the gain in frequency determination was obvious. Including the Hipparcos data enlarged the time basis to about 5000 days for almost all stars. We did no colour transformation for the Hipparcos data, since our method takes possible amplitude differences into account. Since the Hipparcos data are not always as accurate as the ground-based data and 
since the observations sometimes have a rather odd time distribution, there was not always the expected gain in frequency accuracy. In most cases however, the frequency errors became of the order of $10^{-6} \mathrm{~d}^{-1}$.

Amplitude ratios and other characteristics were computed in the different passbands of the Geneva photometric system with frequencies as close as possible to the results of the combined data set. For this purpose we searched again for the closest local minimum in the residuals of the harmonic fit in the Mercator data. In some cases there were differences between the best solution found in the Mercator data alone and this solution. However, differences were small or caused by choosing the wrong alias in the initial solution, as can be seen by comparing Table 1 and Tables 5 to 25 .

\section{Results on the individual stars}

\section{1. $H D 277=H I P 623$}

HD 277 is an F2 star intensively observed by Henry et al. (2001). They found three frequencies and remarked more scatter around light maximum than around light minimum.

The three frequencies found by Henry et al. (2001) were confirmed, although the $2-f$ alias of their third frequency is more significant in the Mercator data. The high significance of this alias is caused by the limited nightly sampling of our data, combined with the length of the period.

The third frequency as derived from the Hipparcos data $\left(1.3437 \mathrm{~d}^{-1}\right)$ deviates from the third frequency $\left(1.3866 \mathrm{~d}^{-1}\right)$ found by Henry et al. (2001) and from our third frequency $\left(1.38709 \mathrm{~d}^{-1}\right)$ as well. At the moment we have no explanation for this, but the standard deviation of the residuals remaining in the Hipparcos data after prewhitening with the first three frequencies is still higher $(0.021 \mathrm{mag})$ than can be expected from the errors on the measurements $(0.017 \mathrm{mag})$. This could indicate that more (low amplitude) frequencies are present, but they could not be detected in the individual data sets. In the combined data set (Hipparcos, Henry et al. 2001, and Mercator data) a fourth frequency at $0.8349 \mathrm{~d}^{-1}$ was detected.

We also observed a few times some extra brightening around light maximum in this star. The standard deviation of the residuals in the Mercator data is about $0.015 \mathrm{mag}$ in $B 1$ and $0.011 \mathrm{mag}$ in $V$. This is an indication of some additional variability.

\section{2. $H D 2842=H I P 2510$}

In previous analyses of the Mercator data (De Ridder et al. 2004; Cuypers et al. 2006) only two frequencies were found, but now the three frequencies, as given by Henry et al. (2005), could be confirmed without any doubt. The three-frequency solution is also clearly present in the Hipparcos data of this star. The frequency given by Koen \& Eyer (2002) for the Hipparcos data $\left(1.78085 \mathrm{~d}^{-1}\right)$ is not present in any of the significant threefrequency solutions.

Fekel et al. (2003) observed no velocity changes in the 2 spectra they obtained, but Mathias et al. (2004) did. The latter also mentioned possible line-profile variations in the blue wing of the lines.

\section{3. $H D 7169=H I P 5674$}

Three frequencies are easily recognized in the Mercator data. Two frequencies are in common with the Henry \& Fekel (2003) results, but their second frequency $\left(2.7640 \mathrm{~d}^{-1}\right)$ is probably an alias of the frequency $1.76031 \mathrm{~d}^{-1}$ found both in the Mercator and Hipparcos data.

This star has 136 Mercator measurements over the period 2001 to 2003, but was unfortunately only once observed in 2004 . Therefore, the errors on the results are larger than usual.

\section{4. $H D 23874=H I P 17826$}

So far only one frequency $\left(2.2565 \mathrm{~d}^{-1}\right)$ has been accepted for this star (Henry \& Fekel 2003), since the second frequency found by Handler (1999) in the Hipparcos data could not be confirmed. However, more than one frequency is expected given the large residuals in all data sets. In the Mercator data a secondary frequency at $2.8883 \mathrm{~d}^{-1}$ or its alias at $1.8856 \mathrm{~d}^{-1}$ is significant. A two-frequency search in the data of Henry \& Fekel (2003) gave also 2.2565 and $1.8849 \mathrm{~d}^{-1}$ as one of the most significant solutions. By combining the Henry \& Fekel data, the Mercator data and the Hipparcos data, the two frequencies could be calculated more precisely.

\subsection{HD $48271=$ HIP $32263=$ V553 Aur}

Handler (1999) found two frequencies for this star in the Hipparcos data: 0.5244 and $0.8688 \mathrm{~d}^{-1}$. Only one frequency is given by Henry \& Fekel (2003): $0.9125 \mathrm{~d}^{-1}$. Martín et al. (2003) suggested the presence of four frequencies in the Hipparcos data. Our analysis confirmd these four frequencies, although for the second frequency an alias is found to be more significant in the Mercator data and the fourth frequency is only marginally significant. The combination of the data sets, with the Hipparcos data included, solves the alias problems and indicates a stable four-frequency solution.

\section{6. $H D 62454=\operatorname{HIP} 37863=D O L y n$}

For this star Kaye et al. (1999) found five frequencies: 1.60146, $1.43678,1.73671,1.83372,1.80753 \mathrm{~d}^{-1}$ in their multi-site campaign of 1997 and 1998 and they suggested one more near $2.9 \mathrm{~d}^{-1}$. The first three have amplitudes larger than $0.01 \mathrm{mag}$ in $V$ and are well identified. The others have smaller amplitudes. With our multifrequency fit techniques, we found in the data of Kaye et al. (1999) the frequencies 1.60139, 1.43675, 1.73637, $1.83373,1.80793 \mathrm{~d}^{-1}$, all very close to the given solution.

The most significant frequency in the Hipparcos data is $1.3329 \mathrm{~d}^{-1}$. This frequency is not related to any of the other frequencies, but this could be due to the odd time distribution of the Hipparcos data.

In the Mercator data of 2001 to 2004 the main frequency found in filters $B$ and $B 1$ is $1.5985 \mathrm{~d}^{-1}$. This could be an alias of the first frequency given by Kaye et al. (1999). In the other filters this frequency cannot unambiguously be identified. A frequency at $1.2032 \mathrm{~d}^{-1}$ is present in all filters.

It seems impossible to connect the frequency solutions of the different campaigns for this star. This could mean that the frequencies and/or the amplitudes in this star are not stable over a longer period or, more likely, that a (different) combination of more frequencies is necessary to describe the data adequately.

The star is a double-lined spectroscopic binary (Kaye 1998) and orbital parameters were given by Kaye et al. (1999) and Mathias et al. (2004). Line profile variations were observed in the primary star by Mathias et al. (2004).

We do not expect a large influence of the secondary component on the pulsational behaviour given the relatively long orbital 

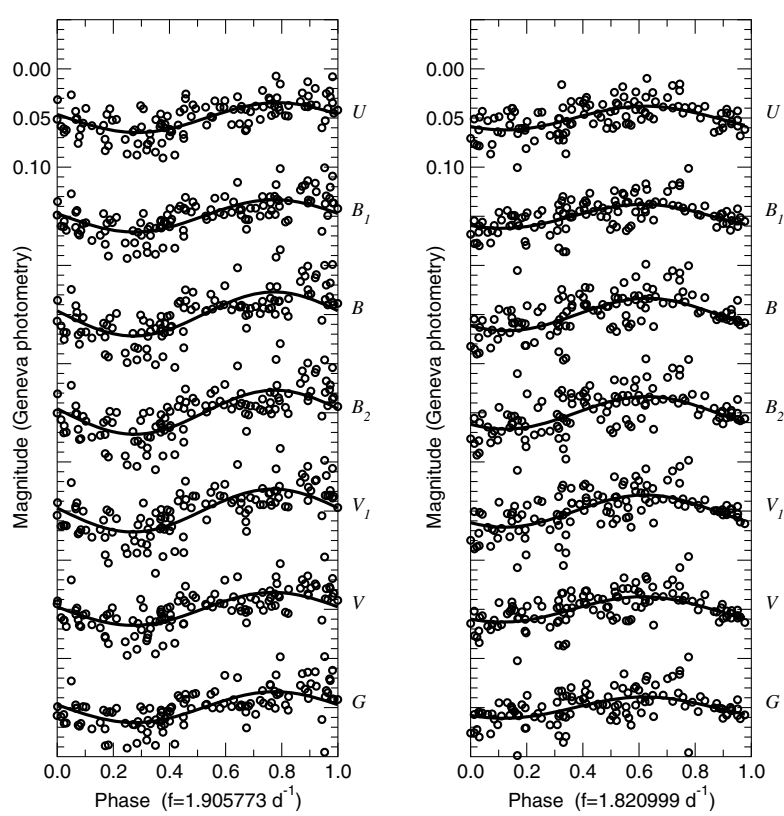

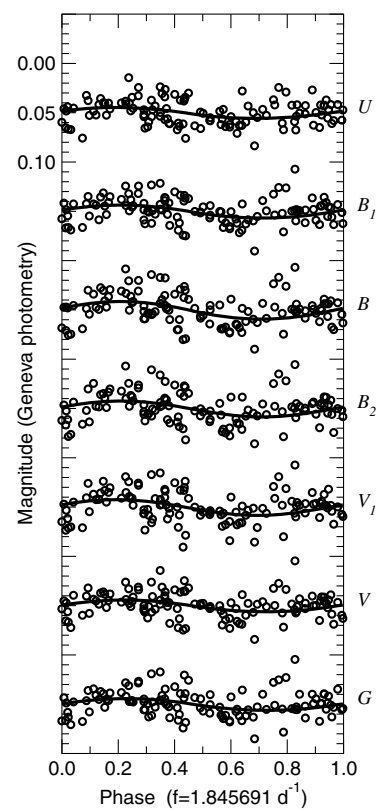

Fig. 1. Phase diagrams of the seven-colour photometry of the newly discovered $\gamma$ Dor star HD 74504 for the indicated frequencies. period of 11.615 days, but currently we have no explanation for the variation of the frequency spectrum from season to season.

\section{7. $H D 69715=$ HIP 40791}

HD 69715 was confirmed as a $\gamma$ Dor star by Henry et al. (2005). The two frequencies found by them, 2.4566 and $2.4416 \mathrm{~d}^{-1}$ with error $0.0002 \mathrm{~d}^{-1}$, are confirmed in the Mercator data, 2.45642 and $2.44120 \mathrm{~d}^{-1}$ with error $0.00007 \mathrm{~d}^{-1}$. In the Hipparcos data a two-frequency solution with 2.0129 and $2.36462 \mathrm{~d}^{-1}$ is present, as already indicated by Martín et al. (2003), but a well defined local minimum is found for 2.45630 and $2.44139 \mathrm{~d}^{-1}$ as well. Therefore, in the combination of all data sets, highly accurate values for these frequencies could be found (errors less than $0.00001 \mathrm{~d}^{-1}$ ). The time span of the observations obtained by Martín et al. (2003) for this star was probably too short to separate these two frequencies. As a consequence, it is not surprising that other values for the frequencies were found, although one of their results, $2.425 \mathrm{~d}^{-1}$, is close to the two frequencies given here. Mathias et al. (2004) observed no line-profile variations in the spectra, pointing to very low velocity amplitudes, probably due to rotational line broadening $\left(v \sin i=145 \mathrm{~km} \mathrm{~s}^{-1}\right)$.

\subsection{HD $74504=$ HIP 43062}

This is a star from the list by Koen \& Eyer (2002) and it is the only star in our sample that is not yet catalogued as variable star. It has all the properties of a $\gamma$ Dor star. The frequency in the Hipparcos data as listed by Koen \& Eyer (2002) is $1.9057 \mathrm{~d}^{-1}$. This frequency $\left(1.90570 \pm 0.00005 \mathrm{~d}^{-1}\right)$ is found easily in the Mercator data, where at least one more frequency could be identified $\left(1.8210 \pm 0.0001 \mathrm{~d}^{-1}\right)$. Although this frequency could be an alias, we prefer this value since a two-frequency analysis of the Hipparcos data gives 1.9058 and $1.8212 \mathrm{~d}^{-1}$ as the best two-frequency solution. In the combined data set we found 1.905773 and $1.820999 \mathrm{~d}^{-1}$. The next frequency candidate here is $1.845691 \mathrm{~d}^{-1}$. Phase diagrams constructed with the frequencies found are given in Fig. 1.

\section{9. $H D 86358=H I P 48895=H R 3936$}

For this $\gamma$ Dor star, identified as such by Handler (1999) and Koen \& Eyer (2002), four frequencies were known from the observations published by Fekel et al. (2003). In the Hipparcos data we identified three of those four frequencies. In the Mercator data the four frequencies (or some clear aliases) are significant as well, but an additional high amplitude variation with frequency $1.00286 \mathrm{~d}^{-1}$ (or an alias near $1.0014 \mathrm{~d}^{-1}$ ) is present. Its amplitude ( $0.022 \mathrm{mag}$ in $V$, but smaller in other filters) is not comparable at all with the little extra power in the periodogram near $1 \mathrm{~d}^{-1}$ mentioned by Fekel et al. (2003) and seems large for a stellar oscillation frequency. It is difficult to explain why a frequency corresponding to one cycle per sidereal day is so significant in this dataset and only marginally or not at all significant in other datasets. It could be an artefact of e.g. imperfect extinction corrections, but in that case we would expect this frequency to be significant in other Mercator data sets as well. This is not the case. After prewhitening with this frequency, it was possible to find a well-defined set of four frequencies in the Mercator data and in the combined data as well. In a five-frequency fit, the variations associated with the frequency near $1 \mathrm{~d}^{-1}$ still have a significant amplitude. Therefore we calculated the associated amplitudes for this frequency as well.

Indications of the binarity of this star were given by Fekel et al. (2003) and Henry \& Fekel (2003). The spectra published by Mathias et al. (2004) clearly showed the binarity and indications of line-profile variations in the slow rotator. Griffin (2006) discussed the system extensively and gave revised orbital elements and mass estimates. In principle, the frequency $1.00286 \mathrm{~d}^{-1}$ or $1.00139 \mathrm{~d}^{-1}$ found in the Mercator data could be a one day alias of a smaller frequency related to the orbital period of this system (33.7 days), but this is unlikely in view of the estimated inclination of $45^{\circ}$ as given by Griffin (2006).

\subsection{HD $100215=$ HIP 56275}

This star is a well known $\gamma$ Dor star. The four frequencies given by Henry \& Fekel (2003), or daily aliases are present in the 
Mercator data as well. The frequencies were refined by combining all data including the Hipparcos data, but some of the results can still be one year aliases of the true frequencies. A candidate for a fifth frequency, present also in the combined data set, is $1.54354 \mathrm{~d}^{-1}$. The star is a binary system of which Griffin (2006) gives high-precision orbital elements.

\subsection{HD $105458=$ HIP 59203}

Henry et al. (2001) found six frequencies in the data for this star. We can easily confirm four of them (or their aliases) in the Mercator data. The six-frequency solution is a local minimum as well and is also present in the combined $V$ data. Therefore, amplitude ratios for all frequencies could be estimated. In the Hipparcos data alone, the frequencies are difficult to find. There $0.7151 \mathrm{~d}^{-1}$ appears as a first frequency, although a frequency of $1.3210 \mathrm{~d}^{-1}$ is present as well.

\subsection{2. $H D 108100=H I P 60571=D D C V n$}

The frequencies found in the Mercator data are compatible with the results of Breger et al. (1997) and Henry \& Fekel (2002). The Hipparcos data are very noisy and could not give independent information. Breger et al. (1997) indicated possible amplitude variations and this might be a reason for not finding the frequencies in a data set with a small number of observations. However, combining the Hipparcos data and the data of Henry \& Fekel (2002) with the Mercator data leads to a well-defined three-frequency solution. Mathias et al. (2004) report that this star is a binary with line-profile variations in the slow rotator.

\subsection{3. $H D 113867=H I P 63951$}

Henry \& Fekel (2003) found five frequencies (0.8887, 2.0084, $1.7785,2.8965$ and $0.8841 \mathrm{~d}^{-1}$ ) and they indicated that there could be some alias problems in their final solution. One of their frequencies is doubtful since it differs only by $0.0046 \mathrm{~d}^{-1}$ from the main frequency and this is at the limit of reliable extraction from an observational campaign of less than 250 days for this star. In the Mercator data a similar solution could be found $\left(0.8884,1.0056,1.7761,1.8910,0.8834 \mathrm{~d}^{-1}\right)$. By combining the data of Henry \& Fekel (2003) with the Mercator data and the Hipparcos observations, some ambiguities regarding day and year aliases could be resolved. A six-frequency solution $\left(0.88838,1.00420,1.77757,1.89378,0.88681,1.42221 \mathrm{~d}^{-1}\right)$ was obtained from this analysis. The sixth frequency (or its alias at $2.42 \mathrm{~d}^{-1}$ ) is also marginally present in Fig. 18 of Henry \& Fekel (2003). The frequency analysis for this star is not decisive. There is one period extremely close to 1 day. The third frequency nearly equals the sum of the first and fifth frequency. As a result not all alias problems nor ambiguities related to possible sum or double frequencies are completely resolved yet. Some indications of closely spaced frequencies near the main frequency are present. The star is very likely to be a binary, as suggested by Henry \& Fekel (2003) and confirmed by Mathias et al. (2004). It is not clear which component is the $\gamma$ Dor star. Since the components are estimated to have spectral type A9 and F1, both stars could be $\gamma$ Dor stars.

\subsection{HD $167858=H I P 89601=H R 6844=$ V2502 Oph}

There is no doubt that this binary star (orbital period: 4.48518 days (Fekel et al. 2003)) is a multiperiodic variable.
Two frequencies (near 0.765 and near $0.697 \mathrm{~d}^{-1}$ ) are significant in all data sets. The third frequency in the Mercator data is near the second frequency cited in Aerts et al. (1998). We identified five frequencies in the combined data, including the third frequency given by Henry \& Fekel (2003). One frequency is close to the $2-f$ alias of the second frequency and this may hamper the frequency analysis of individual ground-based data sets. There is a lot of scatter in the light curves and some excess scatter at maximum brightness.

\subsection{5. $H D 175337=H I P 92837$}

One frequency is clearly present in all the data sets of this star (Hipparcos, Henry et al. 2005, and Mercator data). The Hipparcos data have a few outliers that strongly influence the period search. No secondary frequency could be identified unambiguously.

\subsection{HD $195068=H I P 100859=H R 7828=$ V2121 Cyg}

This star was sometimes observed on the same dates by Henry et al. (2005) and by the Mercator telescope team. The threefrequency solution was easily confirmed in the combined data. A candidate for a fourth frequency occurs near 0.28 or its alias at $1.28 \mathrm{~d}^{-1}$, between $f_{1}$ and $f_{2}$. The first frequency found by Jankov et al. (2006) in spectroscopic data $\left(1.61 \mathrm{~d}^{-1}\right)$ is not significantly present in any of the photometric data sets.

\subsection{7. $H D 206043=H I P 106897=H R 8276=N Z$ Peg}

For this star only three frequencies of the set of five found by Henry et al. (2001) were significant in the Mercator data. However, a local minimum in the residuals exists for the fivefrequency solution both in the Mercator and in the combined data sets. One extra frequency was found in the combined data as well. Also for the six-frequency solution there was convergence to a local minimum in the residuals for the Mercator data. Therefore, amplitudes could be calculated for all six frequencies in the Geneva filters. As expected the amplitudes of frequencies $f_{4}, f_{5}$ en $f_{6}$ are small.

\subsection{HD $207223=H I P 107558=H R 8330=V 372 P e g$}

In the Mercator data the one year alias of the frequency given by Aerts \& Kaye (2001) and Guinan et al. (2001) is found. The Hipparcos data on its own are not very useful to resolve the ambiguity but in the combined data there is a slight preference for the frequency $0.38538 \mathrm{~d}^{-1}$.

\subsection{HD $211699=H I P 110163=P R P e g$}

Only three of the four frequencies (or of their aliases) found by Henry et al. (2007) are significant in the Mercator data. Their second frequency near $1.12 \mathrm{~d}^{-1}$ is not found, but is apparent in the combined data. Furthermore, as already remarked by Henry et al. (2007), their fourth frequency is equal to the difference between $f_{1}$ and $f_{2}$, or, $f_{2}=f_{1}+f_{4}$. Adding a second harmonic to the fourth frequency significantly improves the fit. This adds to the puzzling state of this star as already reported by Mathias et al. (2004), since line-profile variations were observed, but also changes of the equivalent widths of the line. Stellar activity is suggested as an explanation. This could mean that one of the frequencies might be related to rotation. 
Table 1. Results of the frequency analysis of the Mercator data.

\begin{tabular}{|c|c|c|c|c|c|c|c|c|c|c|}
\hline HD number & HIP number & $\begin{array}{l}\text { Spectral } \\
\text { type }\end{array}$ & $N$ & $T(\mathrm{~d})$ & $f_{1}\left(\mathrm{~d}^{-1}\right)$ & $f_{2}\left(\mathrm{~d}^{-1}\right)$ & $f_{3}\left(\mathrm{~d}^{-1}\right)$ & $f_{4}\left(\mathrm{~d}^{-1}\right)$ & $f_{5}\left(\mathrm{~d}^{-1}\right)$ & $f_{6}\left(\mathrm{~d}^{-1}\right)$ \\
\hline 277 & 623 & F0 & 239 & 1148 & 1.11109 & 1.08130 & 1.38709 & & & \\
\hline 2842 & 2510 & F0V & 109 & 1149 & 1.71270 & 1.53729 & 1.67063 & & & \\
\hline 7169 & 5674 & $\mathrm{~F} 2 \mathrm{~V}$ & 96 & 1149 & 1.8225 & 1.9245 & & & & \\
\hline 23874 & 17826 & F0 & 127 & 1045 & 2.2567 & 1.8856 & & & & \\
\hline 48271 & 32263 & F0 & 88 & 869 & 0.9123 & 0.8586 & 0.9314 & 1.7327 & & \\
\hline 62454 & 37863 & F0 & 158 & 847 & 1.5985: & & & & & \\
\hline 69715 & 40791 & A5 & 64 & 805 & 2.45642 & 2.44120 & & & & \\
\hline 74504 & 43062 & F0 & 122 & 794 & 1.90574 & 1.82101 & 1.84570 & & & \\
\hline 86358 & 48895 & $\mathrm{~F} 3 \mathrm{~V}$ & 100 & 805 & 1.1216 & 1.2930 & 1.1869 & 1.1423 & 1.00286 & \\
\hline 100215 & 56275 & Am & 169 & 839 & 1.32191 & 1.42201 & 1.27911 & 1.6159: & & \\
\hline 105458 & 59203 & F0III & 340 & 985 & 1.32084 & 1.25034 & 1.40903 & 0.94631 & 1.55279 & 1.09007 \\
\hline 108100 & 60571 & $\mathrm{~F} 2 \mathrm{~V}$ & 196 & 980 & 1.40132 & 1.32726 & 1.34071: & & & \\
\hline 113867 & 63951 & F0 & 163 & 946 & 0.88841 & 1.00553 & 1.89101 & 1.77613 & 0.88340 & 1.42185 \\
\hline 167858 & 89601 & $\mathrm{~F} 2 \mathrm{~V}$ & 62 & 407 & 0.7650 & 0.6984 & 1.0734 & & & \\
\hline 175337 & 92837 & F5 & 40 & 752 & 1.27109 & & & & & \\
\hline 195068 & 100859 & F5 & 189 & 766 & 1.25054 & 1.29843 & 0.96553 & 0.28517 & & \\
\hline 206043 & 106897 & $\mathrm{~F} 2 \mathrm{~V}$ & 158 & 1163 & 2.4324 & 2.3596 & 2.5243 & & & \\
\hline 207223 & 107558 & F3V & 115 & 1163 & 0.38287 & & & & & \\
\hline 211699 & 110163 & F0 & 97 & 1149 & 0.9327 & 1.1963 & 1.1635 & & & \\
\hline 218396 & 114189 & A5V & 95 & 1151 & 1.9806 & 1.7326 & 0.7676 & & & \\
\hline 221866 & 116434 & $\mathrm{~A} 3 \mathrm{~m}$ & 135 & 1149 & 0.8384 & 0.8772 & 0.8572 & & & \\
\hline
\end{tabular}

The spectral type is from literature. $N$ is the number of observations used, $T$ is the total time span in days. Frequencies not significant in all colour filters are indicated by a colon.

Table 2. Frequencies obtained by combining data sets as described in the text. The definition of the colums is the same as in Table 1.

\begin{tabular}{|c|c|c|c|c|c|c|c|c|c|c|c|}
\hline $\begin{array}{r}\mathrm{HD} \\
\text { number }\end{array}$ & $\begin{array}{r}\text { HIP } \\
\text { number }\end{array}$ & $\begin{array}{l}\text { Spectral } \\
\text { type }\end{array}$ & Source & $N$ & $T(\mathrm{~d})$ & $f_{1}\left(\mathrm{~d}^{-1}\right)$ & $f_{2}\left(\mathrm{~d}^{-1}\right)$ & $f_{3}\left(\mathrm{~d}^{-1}\right)$ & $f_{4}\left(\mathrm{~d}^{-1}\right)$ & $f_{5}\left(\mathrm{~d}^{-1}\right)$ & $f_{6}\left(\mathrm{~d}^{-1}\right)$ \\
\hline 277 & 623 & F0 & HLM & 864 & 5371 & 1.11101 & 1.08099 & 1.38704 & 0.83469 & & \\
\hline 2842 & 2510 & FOV & HLM & 435 & 5377 & 1.71268 & 1.53725 & 1.66799 & & & \\
\hline 7169 & 5674 & $\mathrm{~F} 2 \mathrm{~V}$ & HLM & 548 & 5384 & 1.82259 & 1.92502 & 1.76031 & & & \\
\hline 23874 & 17826 & F0 & HLM & 493 & 5385 & 2.25651 & 1.88562 & & & & \\
\hline 48271 & 32263 & F0 & HLM & 580 & 5109 & 0.91236 & 0.87189 & 0.92314 & 1.75472 & & \\
\hline 62454 & 37863 & F0 & HLM & 264 & 5084 & 1.60156 & 1.43631 & 1.73694 & 1.83413 & 1.81079 & \\
\hline 69715 & 40791 & A5 & HLM & 580 & 4816 & 2.456426 & 2.441492 & & & & \\
\hline 74504 & 43062 & F0 & $\mathrm{H} \mathrm{M}$ & 213 & 5113 & 1.905773 & 1.820999 & 1.845691 & & & \\
\hline 86358 & 48895 & F3V & HLM & 598 & 5169 & 1.289696 & 1.185229 & 1.121666 & 1.142454 & 0.997646 : & \\
\hline 100215 & 56275 & Am & HLM & 946 & 5213 & 1.32188 & 1.42207 & 1.27905 & 1.61480 & 1.54354 & \\
\hline 105458 & 59203 & F0III & HLM & 1043 & 5215 & 1.32083 & 1.25036 & 0.94632 & 1.40903 & 1.09021 & 1.55261 \\
\hline 108100 & 60571 & $\mathrm{~F} 2 \mathrm{~V}$ & HLM & 747 & 5192 & 1.32572 & 1.40136 & 1.36542 & & & \\
\hline 113867 & 63951 & F0 & HLM & 722 & 5180 & 0.88838 & 1.00420 & 1.89379 & 1.77757 & 1.42221 & \\
\hline 167858 & 89601 & $\mathrm{~F} 2 \mathrm{~V}$ & HLM & 622 & 4532 & 0.76508 & 0.69845 & 1.30185 & 1.60558 & 1.07551 & \\
\hline 175337 & 92837 & F5 & HLM & 372 & 4869 & 1.271134 & & & & & \\
\hline 195068 & 100859 & F5 & HLM & 610 & 4994 & 1.250511 & 1.298422 & 0.965416 & 0.284863 & & \\
\hline 206043 & 106897 & $\mathrm{~F} 2 \mathrm{~V}$ & HLM & 600 & 5366 & 2.35944 & 2.43242 & 2.52427 & 2.26551 & 2.59895 & 2.46092 \\
\hline 207223 & 107558 & F3V & $\mathrm{H} \mathrm{M}$ & 204 & 5351 & 0.385383 & & & & & \\
\hline 211699 & 110163 & F0 & HLM & 617 & 5472 & 0.93280 & 1.12646 & 1.16322 & 0.19377 & & \\
\hline 218396 & 114189 & A5V & $\mathrm{H} \mathrm{M}$ & 169 & 5394 & 1.980518 & 1.732528 & 0.767562 & & & \\
\hline 221866 & 116434 & $\mathrm{~A} 3 \mathrm{M}$ & $\mathrm{H} \mathrm{M}$ & 304 & 5394 & 0.877197 & 0.838478 & 1.715664 & & & \\
\hline
\end{tabular}

In the column "source" H means Hipparcos data, M Mercator data and L indicates that other data from literature were used as well. Values in bold indicate newly found frequencies, bold italics means an alias of a previously known frequency was used.

\subsection{0. $H D 218396=H I P 114189=H R 8799=V 342$ Peg}

There is little doubt that this star has at least two frequencies. The first two frequencies $\left(1.9791\right.$ and $1.7368 \mathrm{~d}^{-1}$ ) found in the multisite campaign presented in Zerbi et al. (1999) are present in the Mercator data as well $\left(1.9806\right.$ and $\left.1.7326 \mathrm{~d}^{-1}\right)$. Since their multisite campaign was relatively short, there is agreement within the errors. Combining the data with the Hipparcos measurements reveals another frequency, $0.76762 \mathrm{~d}^{-1}$, which could be an alias of the frequency $f_{4}=0.2479 \mathrm{~d}^{-1}$ of Zerbi et al. (1999).

\subsection{HD $221866=$ HIP 116434}

It is known that for some time series of observations of multiperiodic variations, the technique of consecutive prewhitening will not yield the correct solution (see e.g. Cuypers \& Minarini 1998). The Hipparcos measurements of this star are another 
Table 3. Average magnitudes of the $\gamma$ Dor stars in the Geneva photometric system.

\begin{tabular}{rrrrrrrr}
\hline \hline HD number & $U$ & $B 1$ & $B$ & $B 2$ & $V 1$ & $V$ & $G$ \\
\hline 277 & 9.2075 & 8.7915 & 7.8194 & 9.2134 & 9.0873 & 8.3666 & 9.4687 \\
2842 & 8.7691 & 8.3682 & 7.4092 & 8.8170 & 8.7050 & 7.9858 & 9.0944 \\
7169 & 8.1116 & 7.7034 & 6.7373 & 8.1385 & 8.0028 & 7.2810 & 8.3826 \\
23874 & 9.0657 & 8.6702 & 7.6947 & 9.0866 & 8.9212 & 8.1980 & 9.2914 \\
48271 & 8.2670 & 7.8668 & 6.9094 & 8.3160 & 8.1995 & 7.4807 & 8.5860 \\
62454 & 7.9513 & 7.5519 & 6.5841 & 7.9808 & 7.8535 & 7.1336 & 8.2365 \\
69715 & 7.9669 & 7.5578 & 6.6032 & 8.0114 & 7.8897 & 7.1708 & 8.2751 \\
74504 & 9.6144 & 9.2039 & 8.2523 & 9.6614 & 9.5673 & 8.8519 & 9.9629 \\
86358 & 7.2567 & 6.8881 & 5.9217 & 7.3235 & 7.1841 & 6.4625 & 7.5631 \\
100215 & 8.7886 & 8.3636 & 7.4040 & 8.8074 & 8.6996 & 7.9816 & 9.0866 \\
105458 & 8.5229 & 8.1213 & 7.1688 & 8.5793 & 8.4753 & 7.7584 & 8.8683 \\
108100 & 7.9628 & 7.5625 & 6.5931 & 7.9882 & 7.8371 & 7.1152 & 8.2101 \\
113867 & 7.6159 & 7.1823 & 6.2260 & 7.6367 & 7.5425 & 6.8253 & 7.9382 \\
167858 & 7.4093 & 6.9926 & 6.0277 & 7.4350 & 7.3337 & 6.6143 & 7.7259 \\
175337 & 8.2102 & 7.8103 & 6.8335 & 8.2302 & 8.1012 & 7.3784 & 8.4828 \\
195068 & 6.5117 & 6.0955 & 5.1233 & 6.5300 & 6.4220 & 5.6994 & 6.8119 \\
206043 & 6.5605 & 6.1317 & 5.1674 & 6.5809 & 6.4790 & 5.7572 & 6.8713 \\
207223 & 6.9961 & 6.6026 & 5.6300 & 7.0305 & 6.8966 & 6.1730 & 7.2762 \\
211699 & 9.9568 & 9.5352 & 8.5691 & 9.9679 & 9.8425 & 9.1226 & 10.2255 \\
218396 & 6.6524 & 6.2587 & 5.3186 & 6.7531 & 6.6774 & 5.9601 & 7.0853 \\
221866 & 8.2668 & 7.7669 & 6.8081 & 8.2177 & 8.1670 & 7.4528 & 8.5775 \\
\hline
\end{tabular}

Table 4. Calibration of effective temperature, gravity and metallicity based on the Geneva colours of the $\gamma$ Dor stars. $\Delta$ is the formal calibration error.

\begin{tabular}{rrrrrrr}
\hline \hline HD number & $T_{\text {eff }}(\mathrm{K})$ & $\Delta$ & $\log g$ & $\Delta$ & {$[\mathrm{M} / \mathrm{H}]$} & $\Delta$ \\
\hline 277 & 6995 & 60 & 4.38 & 0.11 & 0.11 & 0.08 \\
2842 & 7091 & 61 & 4.49 & 0.08 & -0.12 & 0.10 \\
7169 & 6905 & 53 & 4.29 & 0.13 & -0.09 & 0.09 \\
23874 & 6720 & 47 & 4.04 & 0.15 & -0.06 & 0.08 \\
48271 & 7050 & 60 & 4.47 & 0.08 & -0.15 & 0.10 \\
62454 & 6997 & 57 & 4.45 & 0.11 & 0.03 & 0.09 \\
69715 & 6987 & 58 & 4.39 & 0.10 & -0.24 & 0.10 \\
74504 & 7221 & 62 & 4.50 & 0.08 & -0.14 & 0.10 \\
86358 & 6910 & 54 & 4.47 & 0.13 & -0.11 & 0.10 \\
100215 & 7109 & 59 & 4.40 & 0.07 & -0.04 & 0.09 \\
105458 & 7144 & 62 & 4.50 & 0.07 & -0.20 & 0.11 \\
108100 & 6809 & 50 & 4.17 & 0.15 & -0.08 & 0.09 \\
113867 & 7195 & 62 & 4.40 & 0.08 & -0.10 & 0.10 \\
167858 & 7177 & 61 & 4.46 & 0.07 & 0.01 & 0.09 \\
175337 & 6993 & 59 & 4.44 & 0.12 & 0.12 & 0.08 \\
195068 & 7133 & 61 & 4.46 & 0.08 & 0.07 & 0.09 \\
206043 & 7144 & 60 & 4.41 & 0.07 & -0.07 & 0.09 \\
207223 & 6952 & 54 & 4.43 & 0.12 & 0.01 & 0.09 \\
211699 & 6984 & 56 & 4.34 & 0.10 & 0.00 & 0.09 \\
218396 & 7355 & 67 & 4.57 & 0.07 & -0.71 & 0.18 \\
221866 & 7480 & 66 & 4.36 & 0.07 & 0.14 & 0.09 \\
\hline
\end{tabular}

example of this phenomenon. In the Hipparcos series a first frequency appears near $0.90 \mathrm{~d}^{-1}$, but only multifrequency fits lead to a solution compatible with the other sets of observations. In the final three-frequency solution, the frequency near $0.90 \mathrm{~d}^{-1}$ is not present anymore. When the Mercator and Hipparcos data were combined (the Henry \& Fekel 2002 data were not used), very precise values for the frequencies could be found (errors less than $0.00001 \mathrm{~d}^{-1}$ ). The frequencies are not independent: in the Mercator data the third frequency is the mean of $f_{1}$ and $f_{2}$, in the data of Henry \& Fekel (2002) and in the Hipparcos data the second frequency is the sum of the two others (within the errors). Adding a harmonic to the third frequency of the Mercator data improves the fit. The two main frequencies are also in agreement with values published by Kaye et al. (2004). Their spectroscopic observations indicate that HD 221866 is a spectroscopic binary with an orbital period of 135 days and a mass ratio of $1.11 \pm 0.03$.

\section{Summary of the results}

The results of the frequency analysis of the Mercator data are summarized in Table 1. For the combined data the frequency solution can be found in Table 2. The errors on the frequencies are of the order of the last decimal given, typically $10^{-5}-10^{-6} \mathrm{~d}^{-1}$.

For all stars with a well-defined frequency solution in the combined data, the amplitudes in the different filters of the Geneva photometric systems were calculated. For each individual star, we give a summary of the results based on the Mercator data in Tables 5 to 25. The errors on the frequencies (and the periods) were calculated with the expressions given by 
Table 5. HD 277.

\begin{tabular}{|c|c|c|c|c|c|c|c|c|c|}
\hline HD 277 & & & $U$ & $B 1$ & $\bar{B}$ & $B 2$ & $V 1$ & $V$ & $G$ \\
\hline & Mean magnitudes: & & 9.2075 & 8.7915 & 7.8194 & 9.2134 & 9.0873 & 8.3666 & 9.4687 \\
\hline & Errors: & & 0.0008 & 0.0009 & 0.0009 & 0.0009 & 0.0008 & 0.0008 & 0.0008 \\
\hline & Amplitude errors: & & 0.0011 & 0.0013 & 0.0013 & 0.0013 & 0.0011 & 0.0011 & 0.0011 \\
\hline HD 277 & Frequency $\left(\mathrm{d}^{-1}\right)$ & $\operatorname{Period}(d)$ & $\bar{U}$ & $B 1$ & $B$ & $B 2$ & $V 1$ & $V$ & $G$ \\
\hline & 1.11111 & 0.90000 & 0.0251 & 0.0359 & 0.0344 & 0.0318 & 0.0259 & 0.0246 & 0.0223 \\
\hline \multirow[t]{3}{*}{ Errors } & 0.00002 & 0.00002 & & & & & & & \\
\hline & Amplitude ratios: & & 0.70 & 1.00 & 0.96 & 0.88 & 0.72 & 0.69 & 0.62 \\
\hline & Errors: & & 0.04 & 0.05 & 0.05 & 0.05 & 0.04 & 0.04 & 0.04 \\
\hline & 1.08135 & 0.92477 & 0.0102 & 0.0134 & 0.0129 & 0.0118 & 0.0095 & 0.0090 & 0.0083 \\
\hline \multirow{3}{*}{ Errors } & 0.00006 & 0.00005 & & & & & & & \\
\hline & Amplitude ratios: & & 0.76 & 1.00 & 0.96 & 0.88 & 0.71 & 0.67 & 0.62 \\
\hline & Errors: & & 0.11 & 0.14 & 0.14 & 0.13 & 0.11 & 0.10 & 0.10 \\
\hline & 1.38708 & 0.72094 & 0.0087 & 0.0131 & 0.0128 & 0.0117 & 0.0090 & 0.0093 & 0.0083 \\
\hline \multirow[t]{3}{*}{ Errors } & 0.00006 & 0.00003 & & & & & & & \\
\hline & Amplitude ratios: & & 0.66 & 1.00 & 0.98 & 0.90 & 0.69 & 0.71 & 0.64 \\
\hline & Errors: & & 0.11 & 0.15 & 0.14 & 0.14 & 0.11 & 0.11 & 0.10 \\
\hline & 0.83461 & 1.19816 & 0.0064 & 0.0076 & 0.0071 & 0.0072 & 0.0061 & 0.0055 & 0.0059 \\
\hline \multirow{3}{*}{ Errors } & 0.00009 & 0.00013 & & & & & & & \\
\hline & Amplitude ratios: & & 0.85 & 1.00 & 0.94 & 0.95 & 0.79 & 0.72 & 0.77 \\
\hline & Errors: & & 0.21 & 0.25 & 0.24 & 0.24 & 0.20 & 0.19 & 0.19 \\
\hline
\end{tabular}

Table 6. HD 2842.

\begin{tabular}{|c|c|c|c|c|c|c|c|c|c|}
\hline HD 2842 & & & $\bar{U}$ & $\overline{B 1}$ & $\bar{B}$ & $\overline{B 2}$ & $V 1$ & $\bar{V}$ & $G$ \\
\hline & Mean magnitudes: & & 8.7691 & 8.3682 & 7.4092 & 8.8170 & 8.7050 & 7.9858 & 9.0944 \\
\hline \multicolumn{3}{|c|}{ Errors: } & 0.0009 & 0.0012 & 0.0011 & 0.0011 & 0.0009 & 0.0008 & 0.0008 \\
\hline \multicolumn{3}{|c|}{ Amplitude errors: } & 0.0013 & 0.0017 & 0.0016 & 0.0015 & 0.0013 & 0.0012 & 0.0012 \\
\hline HD 2842 & Frequency $\left(\mathrm{d}^{-1}\right)$ & $\operatorname{Period}(d)$ & $U$ & $B 1$ & $B$ & $B 2$ & $V 1$ & $V$ & $G$ \\
\hline & 1.71270 & 0.58387 & 0.0244 & 0.0348 & 0.0336 & 0.0317 & 0.0255 & 0.0249 & 0.0229 \\
\hline \multirow[t]{3}{*}{ Errors } & 0.00003 & 0.00001 & & & & & & & \\
\hline & Amplitude ratios: & & 0.70 & 1.00 & 0.97 & 0.91 & 0.73 & 0.71 & 0.66 \\
\hline & Errors: & & 0.05 & 0.07 & 0.06 & 0.06 & 0.05 & 0.05 & 0.05 \\
\hline & 1.53729 & 0.65050 & 0.0137 & 0.0218 & 0.0209 & 0.0194 & 0.0158 & 0.0146 & 0.0140 \\
\hline \multirow[t]{3}{*}{ Errors } & 0.00005 & 0.00002 & & & & & & & \\
\hline & Amplitude ratios: & & 0.63 & 1.00 & 0.96 & 0.89 & 0.72 & 0.67 & 0.64 \\
\hline & Errors: & & 0.08 & 0.11 & 0.10 & 0.10 & 0.08 & 0.08 & 0.07 \\
\hline & 1.67063 & 0.59858 & 0.0089 & 0.0122 & 0.0119 & 0.0117 & 0.0095 & 0.0096 & 0.0092 \\
\hline \multirow[t]{3}{*}{ Errors } & 0.00007 & 0.00003 & & & & & & & \\
\hline & Amplitude ratios: & & 0.73 & 1.00 & 0.98 & 0.96 & 0.78 & 0.79 & 0.75 \\
\hline & Errors: & & 0.15 & 0.19 & 0.19 & 0.18 & 0.15 & 0.15 & 0.14 \\
\hline
\end{tabular}

Table 7. HD 7169.

\begin{tabular}{|c|c|c|c|c|c|c|c|c|c|}
\hline HD 7169 & & & $\overline{\bar{U}}$ & $\overline{\overline{B 1}}$ & $\overline{\bar{B}}$ & $\overline{\overline{B 2}}$ & $\overline{\overline{V 1}}$ & $\overline{\bar{V}}$ & $\overline{\bar{G}}$ \\
\hline & Mean magnitudes: & & 8.1116 & 7.7034 & 6.7373 & 8.1385 & 8.0028 & 7.2810 & 8.3826 \\
\hline & Errors: & & 0.0006 & 0.0006 & 0.0006 & 0.0006 & 0.0006 & 0.0005 & 0.0006 \\
\hline & Amplitude errors: & & 0.0008 & 0.0009 & 0.0008 & 0.0008 & 0.0008 & 0.0007 & 0.0008 \\
\hline HD 7169 & Frequency $\left(\mathrm{d}^{-1}\right)$ & $\operatorname{Period}(d)$ & $\bar{U}$ & $B 1$ & $B$ & $B 2$ & $V 1$ & $\bar{V}$ & $\bar{G}$ \\
\hline & 1.82262 & 0.54866 & 0.0125 & 0.0211 & 0.0195 & 0.0182 & 0.0137 & 0.0138 & 0.0133 \\
\hline Errors & 0.00003 & 0.00001 & & & & & & & \\
\hline & Amplitude ratios: & & 0.59 & 1.00 & 0.92 & 0.86 & 0.65 & 0.66 & 0.63 \\
\hline & Errors: & & 0.05 & 0.06 & 0.06 & 0.05 & 0.05 & 0.04 & 0.04 \\
\hline & 1.92465 & 0.51957 & 0.0057 & 0.0081 & 0.0077 & 0.0075 & 0.0054 & 0.0056 & 0.0050 \\
\hline Errors & 0.00007 & 0.00002 & & & & & & & \\
\hline & Amplitude ratios: & & 0.70 & 1.00 & 0.95 & 0.92 & 0.67 & 0.68 & 0.61 \\
\hline & Errors: & & 0.12 & 0.16 & 0.15 & 0.15 & 0.12 & 0.12 & 0.12 \\
\hline & 1.76050 & 0.56802 & 0.0027 & 0.0049 & 0.0045 & 0.0043 & 0.0033 & 0.0032 & 0.0039 \\
\hline Errors & 0.00014 & 0.00005 & & & & & & & \\
\hline & Amplitude ratios: & & 0.55 & 1.00 & 0.92 & 0.87 & 0.67 & 0.66 & 0.80 \\
\hline & Errors: & & 0.19 & 0.26 & 0.24 & 0.23 & 0.20 & 0.19 & 0.21 \\
\hline
\end{tabular}

Montgomery \& O'Donoghue (1999) or Breger et al. (1999), but only the largest value over the filters is given. In general the difference is less than a factor of 2 . The errors on the amplitudes were calculated with the standard deviations of the final 
Table 8. HD 23874.

\begin{tabular}{|c|c|c|c|c|c|c|c|c|c|}
\hline "HD 23874 & & & $\overline{\bar{U}}$ & $\overline{\overline{B 1}}$ & $\overline{\bar{B}}$ & $\overline{\overline{B 2}}$ & $\overline{\overline{V 1}}$ & $\overline{\bar{V}}$ & $\overline{\bar{G}}$ \\
\hline & Mean magnitudes: & & 9.0657 & 8.6702 & 7.6947 & 9.0866 & 8.9212 & 8.1980 & 9.2914 \\
\hline & Errors: & & 0.0010 & 0.0011 & 0.0008 & 0.0011 & 0.0010 & 0.0008 & 0.0009 \\
\hline & Amplitude errors: & & 0.0014 & 0.0015 & 0.0011 & 0.0015 & 0.0014 & 0.0012 & 0.0013 \\
\hline HD 23874 & Frequency $\left(\mathrm{d}^{-1}\right)$ & $\operatorname{Period}(d)$ & $\bar{U}$ & $B 1$ & $B$ & $B 2$ & $V 1$ & $\vec{V}$ & $G$ \\
\hline & 2.25664 & 0.44314 & 0.0138 & 0.0220 & 0.0216 & 0.0200 & 0.0150 & 0.0147 & 0.0133 \\
\hline \multirow{3}{*}{ Errors } & 0.00005 & 0.00001 & & & & & & & \\
\hline & Amplitude ratios: & & 0.63 & 1.00 & 0.98 & 0.91 & 0.68 & 0.67 & 0.60 \\
\hline & Errors: & & 0.08 & 0.09 & 0.08 & 0.09 & 0.08 & 0.07 & 0.07 \\
\hline \multirow{4}{*}{$\begin{array}{r}f_{2} \\
\text { Errors }\end{array}$} & 1.88559 & 0.53034 & 0.0073 & 0.0092 & 0.0081 & 0.0084 & 0.0060 & 0.0061 & 0.0060 \\
\hline & 0.00012 & 0.00003 & & & & & & & \\
\hline & Amplitude ratios: & & 0.79 & 1.00 & 0.87 & 0.91 & 0.65 & 0.65 & 0.65 \\
\hline & Errors: & & 0.20 & 0.22 & 0.18 & 0.22 & 0.18 & 0.17 & 0.18 \\
\hline
\end{tabular}

Table 9. HD 48271.

\begin{tabular}{|c|c|c|c|c|c|c|c|c|c|}
\hline HD 48271 & & & $\overline{\bar{U}}$ & $\overline{\overline{B 1}}$ & $\overline{\bar{B}}$ & $\overline{\overline{B 2}}$ & $\overline{\overline{V 1}}$ & $\overline{\bar{V}}$ & $\overline{\bar{G}}$ \\
\hline & Mean magnitudes: & & 8.2670 & 7.8668 & 6.9094 & 8.3160 & 8.1995 & 7.4807 & 8.5860 \\
\hline & Errors: & & 0.0009 & 0.0011 & 0.0011 & 0.0011 & 0.0009 & 0.0009 & 0.0008 \\
\hline & Amplitude errors: & & 0.0013 & 0.0016 & 0.0016 & 0.0015 & 0.0013 & 0.0013 & 0.0012 \\
\hline HD 48271 & Frequency $\left(\mathrm{d}^{-1}\right)$ & $\operatorname{Period}(d)$ & $U$ & $B 1$ & $B$ & $B 2$ & $V 1$ & $V$ & $G$ \\
\hline & 0.91236 & 1.09606 & 0.0206 & 0.0288 & 0.0278 & 0.0272 & 0.0212 & 0.0211 & 0.0190 \\
\hline \multirow[t]{3}{*}{ Errors } & 0.00004 & 0.00005 & & & & & & & \\
\hline & Amplitude ratios: & & 0.71 & 1.00 & 0.96 & 0.94 & 0.74 & 0.73 & 0.66 \\
\hline & Errors: & & 0.06 & 0.08 & 0.08 & 0.07 & 0.06 & 0.06 & 0.06 \\
\hline & 0.87189 & 1.14693 & 0.0087 & 0.0111 & 0.0111 & 0.0092 & 0.0080 & 0.0074 & 0.0073 \\
\hline \multirow[t]{3}{*}{ Errors } & 0.00011 & 0.00014 & & & & & & & \\
\hline & Amplitude ratios: & & 0.78 & 1.00 & 1.00 & 0.83 & 0.72 & 0.67 & 0.66 \\
\hline & Errors: & & 0.16 & 0.20 & 0.20 & 0.18 & 0.16 & 0.15 & 0.14 \\
\hline$f_{3}$ & 0.92314 & 1.08326 & 0.0020 & 0.0019 & 0.0019 & 0.0031 & 0.0034 & 0.0039 & 0.0042 \\
\hline \multirow[t]{3}{*}{ Errors } & 0.00053 & 0.00062 & & & & & & & \\
\hline & Amplitude ratios: & & 0.47 & 0.46 & 0.46 & 0.73 & 0.82 & 0.94 & 1.00 \\
\hline & Errors: & & 0.34 & 0.41 & 0.41 & 0.40 & 0.39 & 0.40 & 0.40 \\
\hline \multirow{4}{*}{$\begin{array}{r}f_{4} \\
\text { Errors }\end{array}$} & 1.75472 & 0.56989 & 0.0070 & 0.0103 & 0.0090 & 0.0082 & 0.0058 & 0.0057 & 0.0060 \\
\hline & 0.00015 & 0.00005 & & & & & & & \\
\hline & Amplitude ratios: & & 0.68 & 1.00 & 0.87 & 0.80 & 0.56 & 0.56 & 0.58 \\
\hline & Errors: & & 0.17 & 0.22 & 0.21 & 0.19 & 0.16 & 0.15 & 0.15 \\
\hline
\end{tabular}

Table 10. HD 62454.

\begin{tabular}{|c|c|c|c|c|c|c|c|c|c|}
\hline$\overline{\text { HD } 62454}$ & & & $\bar{U}$ & $\overline{B 1}$ & $\bar{B}$ & $B 2$ & $\overline{V 1}$ & $\bar{V}$ & $G$ \\
\hline & Mean magnitudes: & & 7.9513 & 7.5519 & 6.5841 & 7.9808 & 7.8535 & 7.1336 & 8.2365 \\
\hline & Errors: & & 0.0006 & 0.0006 & 0.0006 & 0.0006 & 0.0006 & 0.0006 & 0.0006 \\
\hline & Amplitude errors: & & 0.0009 & 0.0009 & 0.0009 & 0.0009 & 0.0009 & 0.0008 & 0.0009 \\
\hline HD 62454 & Frequency $\left(\mathrm{d}^{-1}\right)$ & $\operatorname{Period}(d)$ & $U$ & $B 1$ & $B$ & $B 2$ & $V 1$ & $V$ & $G$ \\
\hline & 1.60111 & 0.62457 & 0.0068 & 0.0110 & 0.0099 & 0.0095 & 0.0083 & 0.0077 & 0.0075 \\
\hline Errors & 0.00009 & 0.00003 & & & & & & & \\
\hline & Amplitude ratios: & & 0.62 & 1.00 & 0.90 & 0.87 & 0.76 & 0.70 & 0.69 \\
\hline & Errors: & & 0.10 & 0.12 & 0.11 & 0.11 & 0.10 & 0.09 & 0.10 \\
\hline$f_{2}$ & 1.43619 & 0.69629 & 0.0039 & 0.0072 & 0.0063 & 0.0059 & 0.0049 & 0.0051 & 0.0044 \\
\hline Errors & 0.00015 & 0.00007 & & & & & & & \\
\hline & Amplitude ratios: & & 0.55 & 1.00 & 0.88 & 0.83 & 0.68 & 0.71 & 0.61 \\
\hline & Errors: & & 0.14 & 0.18 & 0.17 & 0.16 & 0.15 & 0.14 & 0.14 \\
\hline & 1.73637 & 0.57591 & 0.0051 & 0.0069 & 0.0066 & 0.0061 & 0.0049 & 0.0049 & 0.0043 \\
\hline Errors & 0.00013 & 0.00004 & & & & & & & \\
\hline & Amplitude ratios: & & 0.74 & 1.00 & 0.96 & 0.88 & 0.71 & 0.70 & 0.62 \\
\hline & Errors: & & 0.16 & 0.19 & 0.18 & 0.17 & 0.16 & 0.15 & 0.15 \\
\hline & 1.83401 & 0.54525 & 0.0050 & 0.0075 & 0.0072 & 0.0067 & 0.0048 & 0.0052 & 0.0042 \\
\hline Errors & 0.00013 & 0.00004 & & & & & & & \\
\hline & Amplitude ratios: & & 0.68 & 1.00 & 0.97 & 0.90 & 0.64 & 0.69 & 0.56 \\
\hline & Errors: & & 0.14 & 0.17 & 0.17 & 0.16 & 0.14 & 0.14 & 0.13 \\
\hline & 1.81124 & 0.55211 & 0.0056 & 0.0067 & 0.0065 & 0.0065 & 0.0045 & 0.0047 & 0.0045 \\
\hline Errors & 0.00013 & 0.00004 & & & & & & & \\
\hline & Amplitude ratios: & & 0.84 & 1.00 & 0.97 & 0.97 & 0.66 & 0.69 & 0.67 \\
\hline & Errors: & & 0.17 & 0.19 & 0.19 & 0.19 & 0.16 & 0.15 & 0.16 \\
\hline
\end{tabular}


Table 11. HD 69715.

\begin{tabular}{|c|c|c|c|c|c|c|c|c|c|}
\hline HD 69715 & & & $\overline{\bar{U}}$ & $\overline{B 1}$ & $\overline{\bar{B}}$ & $\overline{B 2}$ & $\overline{V 1}$ & $\overline{\bar{V}}$ & $\overline{\bar{G}}$ \\
\hline \multicolumn{3}{|c|}{ Mean magnitudes: } & 7.9669 & 7.5578 & 6.6032 & 8.0114 & 7.8897 & 7.1708 & 8.2751 \\
\hline \multicolumn{3}{|c|}{ Errors: } & 0.0006 & 0.0008 & 0.0007 & 0.0007 & 0.0007 & 0.0006 & 0.0007 \\
\hline \multicolumn{3}{|c|}{ Amplitude errors: } & 0.0009 & 0.0011 & 0.0010 & 0.0010 & 0.0010 & 0.0009 & 0.0010 \\
\hline HD 69715 & Frequency $\left(\mathrm{d}^{-1}\right)$ & $\operatorname{Period}(d)$ & $U$ & $B 1$ & $B$ & $B 2$ & $V 1$ & $V$ & $G$ \\
\hline & 2.45643 & 0.40709 & 0.0094 & 0.0134 & 0.0127 & 0.0121 & 0.0091 & 0.0086 & 0.0073 \\
\hline \multirow{3}{*}{ Errors } & 0.00010 & 0.00002 & & & & & & & \\
\hline & Amplitude ratios: & & 0.70 & 1.00 & 0.95 & 0.90 & 0.68 & 0.65 & 0.54 \\
\hline & Errors: & & 0.09 & 0.11 & 0.10 & 0.11 & 0.09 & 0.09 & 0.09 \\
\hline \multirow{4}{*}{$\begin{array}{r}f_{2} \\
\text { Errors }\end{array}$} & 2.44124 & 0.40963 & 0.0092 & 0.0115 & 0.0111 & 0.0101 & 0.0076 & 0.0073 & 0.0064 \\
\hline & 0.00011 & 0.00002 & & & & & & & \\
\hline & Amplitude ratios: & & 0.80 & 1.00 & 0.96 & 0.87 & 0.66 & 0.64 & 0.56 \\
\hline & Errors: & & 0.11 & 0.13 & 0.12 & 0.12 & 0.10 & 0.10 & 0.10 \\
\hline
\end{tabular}

Table 12. HD 74504.

\begin{tabular}{|c|c|c|c|c|c|c|c|c|c|}
\hline HD 74504 & & & $\bar{U}$ & $\overline{B 1}$ & $\bar{B}$ & $B 2$ & $\overline{V 1}$ & $\bar{V}$ & $\bar{G}$ \\
\hline & Mean magnitudes: & & 9.6144 & 9.2039 & 8.2523 & 9.6614 & 9.5673 & 8.8519 & 9.9629 \\
\hline & Errors: & & 0.0011 & 0.0013 & 0.0013 & 0.0013 & 0.0011 & 0.0011 & 0.0011 \\
\hline & Amplitude errors: & & 0.0016 & 0.0019 & 0.0019 & 0.0018 & 0.0016 & 0.0016 & 0.0016 \\
\hline HD 74504 & Frequency $\left(\mathrm{d}^{-1}\right)$ & Period $(d)$ & $U$ & $B 1$ & $B$ & $B 2$ & $V 1$ & $V$ & $G$ \\
\hline & 1.90574 & 0.52473 & 0.0220 & 0.0325 & 0.0326 & 0.0317 & 0.0244 & 0.0239 & 0.0228 \\
\hline Errors & 0.00005 & 0.00001 & & & & & & & \\
\hline & Amplitude ratios: & & 0.68 & 1.00 & 1.00 & 0.97 & 0.75 & 0.73 & 0.70 \\
\hline & Errors: & & 0.06 & 0.08 & 0.08 & 0.08 & 0.06 & 0.06 & 0.06 \\
\hline$f_{2}$ & 1.82101 & 0.54915 & 0.0177 & 0.0241 & 0.0239 & 0.0240 & 0.0191 & 0.0180 & 0.0158 \\
\hline Errors & 0.00007 & 0.00002 & & & & & & & \\
\hline & Amplitude ratios: & & 0.73 & 1.00 & 0.99 & 0.99 & 0.79 & 0.75 & 0.66 \\
\hline & Errors: & & 0.09 & 0.11 & 0.11 & 0.11 & 0.09 & 0.09 & 0.08 \\
\hline & 1.84570 & 0.54180 & 0.0075 & 0.0107 & 0.0121 & 0.0105 & 0.0081 & 0.0090 & 0.0081 \\
\hline Errors & 0.00015 & 0.00004 & & & & & & & \\
\hline & Amplitude ratios: & & 0.62 & 0.88 & 1.00 & 0.87 & 0.67 & 0.74 & 0.67 \\
\hline & Errors: & & 0.17 & 0.21 & 0.22 & 0.20 & 0.16 & 0.17 & 0.17 \\
\hline
\end{tabular}

Table 13. HD 86358.

\begin{tabular}{rrrrrrrrrr}
\hline \hline HD 86358 & & & $U$ & $B 1$ & $B$ & $B 2$ & $V 1$ & $V$ & $G$ \\
\hline & Mean magnitudes: & & 7.2567 & 6.8881 & 5.9217 & 7.3235 & 7.1841 & 6.4625 & 7.5631 \\
& Errors: & & 0.0006 & 0.0006 & 0.0006 & 0.0006 & 0.0006 & 0.0006 & 0.0006 \\
\hline & Amplitude errors: & & 0.0008 & 0.0009 & 0.0009 & 0.0009 & 0.0008 & 0.0008 & 0.0008 \\
\hline HD 86358 & Frequency $\left(\mathrm{d}^{-1}\right)$ & Period $(d)$ & $U$ & $B 1$ & $B$ & $B 2$ & $V 1$ & $V$ & $G$ \\
\hline$f_{1}$ & 1.18526 & 0.84370 & 0.0101 & 0.0158 & 0.0160 & 0.0145 & 0.0105 & 0.0108 & 0.0093 \\
Errors & 0.00006 & 0.00004 & & & & & & & \\
& Amplitude ratios: & & 0.63 & 0.99 & 1.00 & 0.91 & 0.66 & 0.67 & 0.58 \\
& Errors: & & 0.06 & 0.08 & 0.08 & 0.07 & 0.06 & 0.06 & 0.06 \\
\hline$f_{2}$ & 1.28995 & 0.77522 & 0.0102 & 0.0150 & 0.0144 & 0.0135 & 0.0095 & 0.0097 & 0.0084 \\
Errors & 0.00007 & 0.00004 & & & & & & & \\
& Amplitude ratios: & & 0.68 & 1.00 & 0.96 & 0.90 & 0.64 & 0.65 & 0.56 \\
& Errors: & & 0.07 & 0.09 & 0.08 & 0.08 & 0.07 & 0.07 & 0.07 \\
\hline$f_{3}$ & 1.12170 & 0.89150 & 0.0110 & 0.0147 & 0.0147 & 0.0131 & 0.0110 & 0.0110 & 0.0103 \\
Errors & 0.00006 & 0.00004 & & & & & & & \\
& Amplitude ratios: & & 0.75 & 1.00 & 1.00 & 0.89 & 0.75 & 0.75 & 0.70 \\
& Errors: & & 0.07 & 0.09 & 0.09 & 0.08 & 0.07 & 0.07 & 0.07 \\
\hline & & & & & & & & & \\
$f_{4}$ & 1.14247 & 0.87530 & 0.0077 & 0.0097 & 0.0092 & 0.0085 & 0.0071 & 0.0073 & 0.0067 \\
Errors & 0.00008 & 0.00006 & & & & & & & \\
& Amplitude ratios: & & 0.79 & 1.00 & 0.95 & 0.88 & 0.73 & 0.75 & 0.69 \\
& Errors: & & 0.11 & 0.13 & 0.13 & 0.12 & 0.11 & 0.11 & 0.11 \\
\hline$f_{5}$ & 1.00138 & 0.99862 & 0.0160 & 0.0202 & 0.0191 & 0.0207 & 0.0218 & 0.0191 & 0.0218 \\
Errors & 0.00004 & 0.00004 & & & & & & & 1.00 \\
& Amplitude ratios: & & 0.74 & 0.93 & 0.88 & 0.95 & 1.00 & 0.88 & 0.05 \\
\hline & Errors: & & 0.05 & 0.05 & 0.05 & 0.05 & 0.05 & 0.05 & \\
\hline
\end{tabular}


Table 14. HD 100215.

\begin{tabular}{|c|c|c|c|c|c|c|c|c|c|}
\hline \multirow[t]{4}{*}{ HD 100215} & & & $\overline{\bar{U}}$ & $\overline{B B 1}$ & $\bar{B}$ & $\overline{B 2}$ & $\overline{\overline{V 1}}$ & $\overline{\bar{V}}$ & $\bar{G}$ \\
\hline & \multicolumn{2}{|l|}{ Mean magnitudes: } & 8.7886 & 8.3636 & 7.4040 & 8.8074 & 8.6996 & 7.9816 & 9.0866 \\
\hline & \multicolumn{2}{|l|}{ Errors: } & 0.0007 & 0.0008 & 0.0008 & 0.0008 & 0.0007 & 0.0006 & 0.0006 \\
\hline & \multicolumn{2}{|l|}{ Amplitude errors: } & 0.0010 & 0.0012 & 0.0011 & 0.0011 & 0.0010 & 0.0009 & 0.0009 \\
\hline HD 100215 & Frequency $\left(d^{-1}\right)$ & $\operatorname{Period}(d)$ & $\bar{U}$ & $B 1$ & $B$ & $B 2$ & $V 1$ & V & $\bar{G}$ \\
\hline & 1.32188 & 0.75650 & 0.0143 & 0.0229 & 0.0222 & 0.0206 & 0.0167 & 0.0163 & 0.0147 \\
\hline \multirow[t]{3}{*}{ Errors } & 0.00005 & 0.00003 & & & & & & & \\
\hline & \multicolumn{2}{|l|}{ Amplitude ratios: } & 0.63 & 1.00 & 0.97 & 0.90 & 0.73 & 0.71 & 0.64 \\
\hline & \multicolumn{2}{|l|}{ Errors: } & 0.05 & 0.07 & 0.07 & 0.07 & 0.06 & 0.05 & 0.05 \\
\hline & 1.42205 & 0.70321 & 0.0097 & 0.0148 & 0.0142 & 0.0126 & 0.0102 & 0.0100 & 0.0090 \\
\hline \multirow[t]{3}{*}{ Errors } & 0.00007 & 0.00003 & & & & & & & \\
\hline & \multicolumn{2}{|l|}{ Amplitude ratios: } & 0.66 & 1.00 & 0.95 & 0.85 & 0.69 & 0.67 & 0.61 \\
\hline & \multicolumn{2}{|l|}{ Errors: } & 0.09 & 0.11 & 0.10 & 0.10 & 0.09 & 0.08 & 0.08 \\
\hline \multirow{4}{*}{$\begin{array}{r}f_{3} \\
\text { Errors }\end{array}$} & 1.27908 & 0.78181 & 0.0058 & 0.0084 & 0.0085 & 0.0080 & 0.0067 & 0.0069 & 0.0065 \\
\hline & 0.00011 & 0.00007 & & & & & & & \\
\hline & \multicolumn{2}{|l|}{ Amplitude ratios: } & 0.68 & 1.00 & 1.00 & 0.94 & 0.79 & 0.82 & 0.77 \\
\hline & \multicolumn{2}{|l|}{ Errors: } & 0.15 & 0.19 & 0.18 & 0.18 & 0.16 & 0.15 & 0.15 \\
\hline \multirow{4}{*}{$\begin{array}{r}f_{4} \\
\text { Errors }\end{array}$} & 1.61592 & 0.61884 & 0.0036 & 0.0065 & 0.0056 & 0.0055 & 0.0039 & 0.0042 & 0.0042 \\
\hline & 0.00018 & 0.00007 & & & & & & & \\
\hline & \multicolumn{2}{|l|}{ Amplitude ratios: } & 0.55 & 1.00 & 0.87 & 0.85 & 0.60 & 0.64 & 0.65 \\
\hline & \multicolumn{2}{|l|}{ Errors: } & 0.18 & 0.25 & 0.23 & 0.23 & 0.19 & 0.18 & 0.19 \\
\hline \multirow{4}{*}{$\begin{array}{r}f_{5} \\
\text { Errors }\end{array}$} & 1.54452 & 0.64745 & 0.0052 & 0.0072 & 0.0070 & 0.0070 & 0.0053 & 0.0050 & 0.0057 \\
\hline & 0.00013 & 0.00005 & & & & & & & \\
\hline & \multicolumn{2}{|l|}{ Amplitude ratios: } & 0.72 & 1.00 & 0.97 & 0.98 & 0.73 & 0.70 & 0.79 \\
\hline & \multicolumn{2}{|l|}{ Errors: } & 0.18 & 0.23 & 0.22 & 0.22 & 0.18 & 0.17 & 0.18 \\
\hline
\end{tabular}

Table 15. HD 105458.

\begin{tabular}{|c|c|c|c|c|c|c|c|c|c|}
\hline \multirow[t]{4}{*}{ "HD 105458} & & & $\bar{U}$ & $B 1$ & $\bar{B}$ & $\overline{B 2}$ & $\overline{V 1}$ & $\bar{V}$ & $\bar{G}$ \\
\hline & \multicolumn{2}{|l|}{ Mean magnitudes: } & 8.5229 & 8.1213 & 7.1688 & 8.5793 & 8.4753 & 7.7584 & 8.8683 \\
\hline & Errors: & & 0.0009 & 0.0012 & 0.0011 & 0.0011 & 0.0010 & 0.0009 & 0.0008 \\
\hline & \multicolumn{2}{|l|}{ Amplitude errors: } & 0.0011 & 0.0014 & 0.0013 & 0.0013 & 0.0011 & 0.0011 & 0.0010 \\
\hline HD 105458 & Frequency $\left(\mathrm{d}^{-1}\right)$ & Period $(d)$ & $U$ & $B 1$ & $B$ & $B 2$ & $V 1$ & $V$ & $G$ \\
\hline & 1.32084 & 0.75709 & 0.0196 & 0.0309 & 0.0292 & 0.0288 & 0.0223 & 0.0218 & 0.0205 \\
\hline \multirow[t]{3}{*}{ Errors } & 0.00003 & 0.00002 & & & & & & & \\
\hline & \multicolumn{2}{|l|}{ Amplitude ratios: } & 0.63 & 1.00 & 0.94 & 0.93 & 0.72 & 0.71 & 0.67 \\
\hline & & 0.05 & 0.06 & 0.06 & 0.06 & 0.05 & 0.05 & 0.04 \\
\hline & 1.09007 & 0.91737 & 0.0117 & 0.0154 & 0.0151 & 0.0155 & 0.0125 & 0.0120 & 0.0106 \\
\hline \multirow[t]{3}{*}{ Errors } & 0.00006 & 0.00005 & & & & & & & \\
\hline & \multicolumn{2}{|l|}{$\begin{array}{l}\text { Amplitude ratios: } \\
\text { Errors: }\end{array}$} & 0.76 & 0.99 & 0.98 & 1.00 & 0.80 & 0.77 & 0.69 \\
\hline & Errors: & & 0.10 & 0.12 & 0.12 & 0.12 & 0.10 & 0.09 & 0.09 \\
\hline \multirow{4}{*}{$\begin{array}{r}f_{3} \\
\text { Errors }\end{array}$} & 1.25034 & 0.79978 & 0.0103 & 0.0151 & 0.0146 & 0.0139 & 0.0108 & 0.0109 & 0.0105 \\
\hline & 0.00006 & 0.00004 & & & & & & & \\
\hline & \multicolumn{2}{|l|}{ Amplitude ratios: } & 0.68 & 1.00 & 0.97 & 0.92 & 0.72 & 0.72 & 0.70 \\
\hline & \multicolumn{2}{|l|}{ Errors: } & 0.10 & 0.13 & 0.12 & 0.12 & 0.10 & 0.10 & 0.09 \\
\hline \multirow{4}{*}{$\begin{array}{r}f_{4} \\
\text { Errors }\end{array}$} & 1.40906 & 0.70969 & 0.0083 & 0.0120 & 0.0114 & 0.0113 & 0.0088 & 0.0088 & 0.0080 \\
\hline & 0.00007 & 0.00004 & & & & & & & \\
\hline & \multicolumn{2}{|l|}{ Amplitude ratios: } & 0.70 & 1.00 & 0.95 & 0.94 & 0.74 & 0.73 & 0.67 \\
\hline & \multicolumn{2}{|l|}{ Errors: } & 0.12 & 0.16 & 0.15 & 0.15 & 0.13 & 0.12 & 0.12 \\
\hline & 0.94631 & 1.05674 & 0.0085 & 0.0114 & 0.0114 & 0.0097 & 0.0083 & 0.0081 & 0.0073 \\
\hline \multirow[t]{3}{*}{ Errors } & 0.00008 & 0.00009 & & & & & & & \\
\hline & \multicolumn{2}{|l|}{ Amplitude ratios: } & 0.75 & 1.00 & 1.00 & 0.85 & 0.73 & 0.71 & 0.64 \\
\hline & \multicolumn{2}{|l|}{ Errors: } & 0.13 & 0.17 & 0.16 & 0.15 & 0.13 & 0.12 & 0.12 \\
\hline \multirow{4}{*}{$\begin{array}{r}f_{6} \\
\text { Errors }\end{array}$} & 1.55279 & 0.64400 & 0.0066 & 0.0097 & 0.0095 & 0.0090 & 0.0078 & 0.0074 & 0.0065 \\
\hline & 0.00010 & 0.00004 & & & & & & & \\
\hline & \multicolumn{2}{|l|}{ Amplitude ratios: } & 0.68 & 1.00 & 0.98 & 0.93 & 0.80 & 0.76 & 0.67 \\
\hline & \multicolumn{2}{|l|}{ Errors: } & 0.15 & 0.20 & 0.19 & 0.19 & 0.16 & 0.15 & 0.14 \\
\hline
\end{tabular}

residuals. As a consequence, the errors are the same for each frequency in a multifrequency solution. Since these values are larger than the formal values obtained from a harmonic leastsquares fit, they can be considered as a reliable upper limit. The relative errors will increase and this is reflected in the increase of errors on the amplitude ratios from the dominant to the lower amplitude modes (see Fig. 2).

The mean values of the magnitudes in each filter as obtained from the fit (with errors) are given in each table as well. The values were used for the calibration of effective temperature, gravity 
Table 16. HD 108100.

\begin{tabular}{|c|c|c|c|c|c|c|c|c|c|}
\hline$\overline{\text { HD } 108100}$ & & & $\overline{\bar{U}}$ & $\overline{B 1}$ & $\bar{B}$ & $\overline{B 2}$ & $\overline{\overline{V 1}}$ & $\overline{\bar{V}}$ & $\overline{\bar{G}}$ \\
\hline & Mean magnitudes: & & 7.9628 & 7.5625 & 6.5931 & 7.9882 & 7.8371 & 7.1152 & 8.2101 \\
\hline & Errors: & & 0.0007 & 0.0008 & 0.0008 & 0.0007 & 0.0006 & 0.0006 & 0.0006 \\
\hline & Amplitude errors: & & 0.0010 & 0.0011 & 0.0011 & 0.0010 & 0.0009 & 0.0009 & 0.0009 \\
\hline HD 108100 & Frequency $\left(\mathrm{d}^{-1}\right)$ & $\operatorname{Period}(d)$ & $U$ & $B 1$ & $B$ & $B 2$ & $V 1$ & $V$ & $G$ \\
\hline & 1.40134 & 0.71360 & 0.0076 & 0.0124 & 0.0118 & 0.0109 & 0.0084 & 0.0085 & 0.0074 \\
\hline \multirow[t]{3}{*}{ Errors } & 0.00007 & 0.00004 & & & & & & & \\
\hline & Amplitude ratios: & & 0.61 & 1.00 & 0.95 & 0.88 & 0.68 & 0.69 & 0.60 \\
\hline & Errors: & & 0.09 & 0.13 & 0.12 & 0.12 & 0.10 & 0.09 & 0.09 \\
\hline \multirow{4}{*}{$\begin{array}{r}f_{2} \\
\text { Errors }\end{array}$} & 1.32572 & 0.75431 & 0.0064 & 0.0104 & 0.0098 & 0.0091 & 0.0072 & 0.0065 & 0.0061 \\
\hline & 0.00008 & 0.00005 & & & & & & & \\
\hline & Amplitude ratios: & & 0.61 & 1.00 & 0.94 & 0.88 & 0.69 & 0.63 & 0.59 \\
\hline & Errors: & & 0.11 & 0.15 & 0.14 & 0.14 & 0.12 & 0.11 & 0.11 \\
\hline \multirow{4}{*}{$\begin{array}{r}f_{3} \\
\text { Errors }\end{array}$} & 1.36559 & 0.73228 & 0.0039 & 0.0047 & 0.0045 & 0.0049 & 0.0036 & 0.0032 & 0.0029 \\
\hline & 0.00017 & 0.00009 & & & & & & & \\
\hline & Amplitude ratios: & & 0.81 & 0.96 & 0.93 & 1.00 & 0.74 & 0.65 & 0.60 \\
\hline & Errors: & & 0.26 & 0.30 & 0.29 & 0.30 & 0.25 & 0.23 & 0.22 \\
\hline
\end{tabular}

Table 17. HD 113867.

\begin{tabular}{|c|c|c|c|c|c|c|c|c|c|}
\hline HD 113867 & & & $\overline{\bar{U}}$ & $\overline{B 1}$ & $\overline{\bar{B}}$ & $\overline{B 2}$ & $\overline{\overline{V 1}}$ & $\overline{\bar{V}}$ & $\overline{\bar{G}}$ \\
\hline & Mean magnitudes: & & 7.6159 & 7.1823 & 6.2260 & 7.6367 & 7.5425 & 6.8253 & 7.9382 \\
\hline & Errors: & & 0.0007 & 0.0007 & 0.0006 & 0.0006 & 0.0006 & 0.0005 & 0.0006 \\
\hline & Amplitude errors: & & 0.0010 & 0.0010 & 0.0009 & 0.0009 & 0.0008 & 0.0007 & 0.0008 \\
\hline HD 113867 & Frequency $\left(\mathrm{d}^{-1}\right)$ & $\operatorname{Period}(d)$ & $U$ & $B 1$ & $B$ & $B 2$ & $V 1$ & $V$ & $G$ \\
\hline & 0.88841 & 1.12561 & 0.0332 & 0.0429 & 0.0426 & 0.0400 & 0.0320 & 0.0312 & 0.0282 \\
\hline \multirow[t]{3}{*}{ Errors } & 0.00002 & 0.00002 & & & & & & & \\
\hline & Amplitude ratios: & & 0.77 & 1.00 & 0.99 & 0.93 & 0.75 & 0.73 & 0.66 \\
\hline & Errors: & & 0.03 & 0.03 & 0.03 & 0.03 & 0.02 & 0.02 & 0.02 \\
\hline & 1.00553 & 0.99450 & 0.0189 & 0.0223 & 0.0224 & 0.0212 & 0.0190 & 0.0192 & 0.0165 \\
\hline \multirow[t]{3}{*}{ Errors } & 0.00003 & 0.00003 & & & & & & & \\
\hline & Amplitude ratios: & & 0.84 & 0.99 & 1.00 & 0.94 & 0.85 & 0.86 & 0.74 \\
\hline & Errors: & & 0.06 & 0.06 & 0.06 & 0.05 & 0.05 & 0.05 & 0.04 \\
\hline \multirow{4}{*}{$\begin{array}{r}f_{3} \\
\text { Errors }\end{array}$} & 1.89101 & 0.52882 & 0.0189 & 0.0214 & 0.0225 & 0.0211 & 0.0162 & 0.0146 & 0.0144 \\
\hline & 0.00003 & 0.00001 & & & & & & & \\
\hline & Amplitude ratios: & & 0.84 & 0.95 & 1.00 & 0.94 & 0.72 & 0.65 & 0.64 \\
\hline & Errors: & & 0.06 & 0.06 & 0.06 & 0.05 & 0.04 & 0.04 & 0.04 \\
\hline \multirow{4}{*}{$\begin{array}{r}J_{4} \\
\text { Errors }\end{array}$} & 1.77613 & 0.56302 & 0.0099 & 0.0123 & 0.0120 & 0.0120 & 0.0090 & 0.0089 & 0.0080 \\
\hline & 0.00006 & 0.00002 & & & & & & & \\
\hline & Amplitude ratios: & & 0.80 & 1.00 & 0.97 & 0.97 & 0.73 & 0.72 & 0.65 \\
\hline & Errors: & & 0.10 & 0.11 & 0.11 & 0.10 & 0.08 & 0.08 & 0.08 \\
\hline \multirow{4}{*}{$\begin{array}{r}f_{5} \\
\text { Errors }\end{array}$} & 0.88340 & 1.13199 & 0.0035 & 0.0058 & 0.0047 & 0.0055 & 0.0037 & 0.0030 & 0.0032 \\
\hline & 0.00017 & 0.00021 & & & & & & & \\
\hline & Amplitude ratios: & & 0.61 & 1.00 & 0.81 & 0.96 & 0.65 & 0.51 & 0.55 \\
\hline & Errors: & & 0.20 & 0.24 & 0.21 & 0.22 & 0.17 & 0.16 & 0.16 \\
\hline \multirow{4}{*}{$\begin{array}{r}f_{6} \\
\text { Errors }\end{array}$} & 1.42185 & 0.70331 & 0.0054 & 0.0068 & 0.0070 & 0.0065 & 0.0061 & 0.0061 & 0.0057 \\
\hline & 0.00011 & 0.00005 & & & & & & & \\
\hline & Amplitude ratios: & & 0.77 & 0.97 & 1.00 & 0.93 & 0.86 & 0.87 & 0.81 \\
\hline & Errors: & & 0.18 & 0.18 & 0.18 & 0.17 & 0.16 & 0.15 & 0.15 \\
\hline
\end{tabular}

and a metallicity indicator according to Kunzli et al. (1997), of which a summary is given in Table 4.

\section{Conclusions}

Almost all frequencies of known $\gamma$ Dor stars presented in this paper are confirmed and one candidate (HD 74504) can now be considered as an additional member of this class. By combining the Mercator data with data available from the literature (including Hipparcos data), sets of frequencies were enlarged, confirmed and/or more precisely determined. Many $\gamma$ Dor stars have frequencies that are very stable over a long period (1989-2004). Only in a few cases did the frequencies found in the Hipparcos data (1989-1993) deviate from the values found in more recent data. Differences are only a few hundredths of a cycle per day in some cases and yearly aliasing could be the cause, but in a few other stars there was no agreement at all. This may be caused by the coarse sampling of the Hipparcos data, but it could also be an indication of some more hidden frequencies, complex beating patterns and/or changing frequencies.

For the large majority of the $\gamma$ Dor stars the behaviour of the amplitude ratios as a function of the wavelength is very similar. We will explore these observational results in future modelling of their oscillations, with the goal to check if we can constrain the input physics of the models for F-type stars. Our study is the only consistent long-term multicolour photometric database of 
Table 18. HD 167858.

\begin{tabular}{|c|c|c|c|c|c|c|c|c|c|}
\hline \multirow[t]{4}{*}{ HD 167858} & & & $\bar{U}$ & $\overline{B 1}$ & $\bar{B}$ & $\overline{B 2}$ & $\overline{\overline{V 1}}$ & $\bar{V}$ & $\overline{\bar{G}}$ \\
\hline & Mean magnitudes: & & 7.4093 & 6.9926 & 6.0277 & 7.4350 & 7.3337 & 6.6143 & 7.7259 \\
\hline & Errors: & & 0.0011 & 0.0014 & 0.0014 & 0.0013 & 0.0011 & 0.0011 & 0.0011 \\
\hline & Amplitude errors: & & 0.0016 & 0.0020 & 0.0020 & 0.0019 & 0.0015 & 0.0015 & 0.0015 \\
\hline \multirow{5}{*}{$\begin{array}{r}\text { HD } 167858 \\
\text { Errors }\end{array}$} & Frequency $\left(\mathrm{d}^{-1}\right)$ & $\operatorname{Period}(d)$ & $\bar{U}$ & $B 1$ & $B$ & $B 2$ & $V 1$ & $\bar{V}$ & $\bar{G}$ \\
\hline & 0.76504 & 1.30712 & 0.0295 & 0.0412 & 0.0394 & 0.0363 & 0.0281 & 0.0288 & 0.0255 \\
\hline & 0.00008 & 0.00013 & & & & & & & \\
\hline & Amplitude ratios: & & 0.72 & 1.00 & 0.96 & 0.88 & 0.68 & 0.70 & 0.62 \\
\hline & Errors: & & 0.05 & 0.07 & 0.07 & 0.06 & 0.05 & 0.05 & 0.05 \\
\hline \multirow{4}{*}{$\begin{array}{r}f_{2} \\
\text { Errors }\end{array}$} & 0.69543 & 1.43796 & 0.0217 & 0.0300 & 0.0283 & 0.0264 & 0.0207 & 0.0212 & 0.0181 \\
\hline & 0.00011 & 0.00023 & & & & & & & \\
\hline & Amplitude ratios: & & 0.72 & 1.00 & 0.94 & 0.88 & 0.69 & 0.70 & 0.60 \\
\hline & Errors: & & 0.07 & 0.10 & 0.09 & 0.09 & 0.07 & 0.07 & 0.06 \\
\hline \multirow{4}{*}{$\begin{array}{r}f_{3} \\
\text { Errors }\end{array}$} & 1.07613 & 0.92926 & 0.0142 & 0.0189 & 0.0183 & 0.0174 & 0.0134 & 0.0133 & 0.0131 \\
\hline & 0.00016 & 0.00014 & & & & & & & \\
\hline & Amplitude ratios: & & 0.75 & 1.00 & 0.97 & 0.92 & 0.71 & 0.70 & 0.69 \\
\hline & Errors: & & 0.12 & 0.15 & 0.15 & 0.14 & 0.11 & 0.11 & 0.11 \\
\hline \multirow{4}{*}{$\begin{array}{r}f_{4} \\
\text { Errors }\end{array}$} & 1.30172 & 0.76821 & 0.0101 & 0.0143 & 0.0138 & 0.0130 & 0.0106 & 0.0101 & 0.0097 \\
\hline & 0.00022 & 0.00013 & & & & & & & \\
\hline & Amplitude ratios: & & 0.70 & 1.00 & 0.97 & 0.91 & 0.74 & 0.71 & 0.68 \\
\hline & Errors: & & 0.15 & 0.20 & 0.20 & 0.19 & 0.15 & 0.15 & 0.14 \\
\hline \multirow{4}{*}{$\begin{array}{r}f_{5} \\
\text { Errors }\end{array}$} & 1.60489 & 0.62310 & 0.0053 & 0.0074 & 0.0074 & 0.0058 & 0.0049 & 0.0058 & 0.0042 \\
\hline & 0.00047 & 0.00018 & & & & & & & \\
\hline & Amplitude ratios: & & 0.71 & 1.00 & 1.00 & 0.79 & 0.66 & 0.78 & 0.57 \\
\hline & Errors: & & 0.29 & 0.38 & 0.38 & 0.34 & 0.27 & 0.30 & 0.25 \\
\hline
\end{tabular}

Table 19. HD 175337.

\begin{tabular}{|c|c|c|c|c|c|c|c|c|c|}
\hline HD 175337 & & & $\overline{\bar{U}}$ & $\overline{\overline{B 1}}$ & $\overline{\bar{B}}$ & $\overline{\bar{B} 2}$ & $\overline{\overline{V 1}}$ & $\overline{\bar{V}}$ & $\overline{\bar{G}}$ \\
\hline & Mean magnitudes: & & 8.2102 & 7.8103 & 6.8335 & 8.2302 & 8.1012 & 7.3784 & 8.4828 \\
\hline & Errors: & & 0.0011 & 0.0011 & 0.0011 & 0.0009 & 0.0009 & 0.0008 & 0.0010 \\
\hline & Amplitude errors: & & 0.0016 & 0.0016 & 0.0016 & 0.0013 & 0.0013 & 0.0011 & 0.0014 \\
\hline HD 175337 & Frequency $\left(\mathrm{d}^{-1}\right)$ & $\operatorname{Period}(d)$ & $\bar{U}$ & $B 1$ & $B$ & $B 2$ & $V 1$ & $V$ & $G$ \\
\hline \multirow{4}{*}{$\begin{array}{r}f_{1} \\
\text { Errors }\end{array}$} & 1.27109 & 0.78673 & 0.0154 & 0.0215 & 0.0206 & 0.0195 & 0.0153 & 0.0143 & 0.0145 \\
\hline & 0.00007 & 0.00005 & & & & & & & \\
\hline & Amplitude ratios: & & 0.72 & 1.00 & 0.96 & 0.91 & 0.71 & 0.67 & 0.68 \\
\hline & Errors: & & 0.09 & 0.11 & 0.10 & 0.09 & 0.08 & 0.07 & 0.08 \\
\hline
\end{tabular}

Table 20. HD 195068.

\begin{tabular}{|c|c|c|c|c|c|c|c|c|c|}
\hline HD 195068 & & & $\overline{\bar{U}}$ & $\overline{\overline{B 1}}$ & $\overline{\bar{B}}$ & $\overline{\overline{B 2}}$ & $\overline{\overline{V 1}}$ & $\overline{\bar{V}}$ & $\overline{\bar{G}}$ \\
\hline & Mean magnitudes: & & 6.5117 & 6.0955 & 5.1233 & 6.5300 & 6.4220 & 5.6994 & 6.8119 \\
\hline & Errors: & & 0.0008 & 0.0011 & 0.0011 & 0.0011 & 0.0008 & 0.0008 & 0.0008 \\
\hline & Amplitude errors: & & 0.0011 & 0.0016 & 0.0015 & 0.0015 & 0.0012 & 0.0012 & 0.0011 \\
\hline HD 195068 & Frequency $\left(\mathrm{d}^{-1}\right)$ & $\operatorname{Period}(d)$ & $\bar{U}$ & $B 1$ & $B$ & $B 2$ & $V 1$ & $\bar{V}$ & $G$ \\
\hline & 1.25054 & 0.79965 & 0.0415 & 0.0603 & 0.0576 & 0.0546 & 0.0428 & 0.0413 & 0.0382 \\
\hline \multirow[t]{3}{*}{ Errors } & 0.00002 & 0.00001 & & & & & & & \\
\hline & Amplitude ratios: & & 0.69 & 1.00 & 0.95 & 0.90 & 0.71 & 0.69 & 0.63 \\
\hline & Errors: & & 0.03 & 0.04 & 0.04 & 0.03 & 0.03 & 0.03 & 0.02 \\
\hline \multirow{4}{*}{$\begin{array}{r}f_{2} \\
\text { Errors }\end{array}$} & 1.29843 & 0.77016 & 0.0199 & 0.0285 & 0.0272 & 0.0262 & 0.0206 & 0.0202 & 0.0182 \\
\hline & 0.00005 & 0.00003 & & & & & & & \\
\hline & Amplitude ratios: & & 0.70 & 1.00 & 0.95 & 0.92 & 0.72 & 0.71 & 0.64 \\
\hline & Errors: & & 0.06 & 0.08 & 0.07 & 0.07 & 0.06 & 0.06 & 0.05 \\
\hline \multirow{4}{*}{$\begin{array}{r}f_{3} \\
\text { Errors }\end{array}$} & 0.96553 & 1.03570 & 0.0163 & 0.0221 & 0.0216 & 0.0205 & 0.0164 & 0.0158 & 0.0148 \\
\hline & 0.00006 & 0.00006 & & & & & & & \\
\hline & Amplitude ratios: & & 0.73 & 1.00 & 0.97 & 0.93 & 0.74 & 0.71 & 0.67 \\
\hline & Errors: & & 0.07 & 0.10 & 0.10 & 0.09 & 0.07 & 0.07 & 0.07 \\
\hline \multirow{4}{*}{$\begin{array}{r}f_{4} \\
\text { Errors }\end{array}$} & 0.28517 & 3.50668 & 0.0120 & 0.0160 & 0.0157 & 0.0145 & 0.0120 & 0.0115 & 0.0108 \\
\hline & 0.00007 & 0.00092 & & & & & & & \\
\hline & Amplitude ratios: & & 0.75 & 1.00 & 0.98 & 0.91 & 0.75 & 0.72 & 0.68 \\
\hline & Errors: & & 0.10 & 0.14 & 0.13 & 0.13 & 0.10 & 0.10 & 0.10 \\
\hline
\end{tabular}


Table 21. HD 206043.

\begin{tabular}{rrrrrrrrrr}
\hline \hline HD 206043 & & & $U$ & $B 1$ & $B$ & $B 2$ & $V 1$ & $V$ & $G$ \\
\hline & Mean magnitudes: & & 6.5605 & 6.1317 & 5.1674 & 6.5809 & 6.4790 & 5.7572 & 6.8713 \\
& Errors: & & 0.0007 & 0.0007 & 0.0007 & 0.0007 & 0.0007 & 0.0007 & 0.0007 \\
\hline & Amplitude errors: & & 0.0010 & 0.0010 & 0.0010 & 0.0010 & 0.0010 & 0.0010 & 0.0010 \\
\hline HD 206043 & Frequency $\left(\mathrm{d}^{-1}\right)$ & Period $(d)$ & $U$ & $B 1$ & $B$ & $B 2$ & $V 1$ & $V$ & $G$ \\
\hline$f_{1}$ & 2.35951 & 0.42382 & 0.0114 & 0.0172 & 0.0166 & 0.0156 & 0.0129 & 0.0126 & 0.0118 \\
Errors & 0.00004 & 0.00001 & & & & & & & \\
& Amplitude ratios: & & 0.66 & 1.00 & 0.97 & 0.91 & 0.75 & 0.73 & 0.69 \\
& Errors: & & 0.07 & 0.08 & 0.08 & 0.08 & 0.07 & 0.07 & 0.07 \\
\hline$f_{2}$ & 2.43246 & 0.41111 & 0.0124 & 0.0177 & 0.0167 & 0.0158 & 0.0134 & 0.0130 & 0.0119 \\
Errors & 0.00004 & 0.00001 & & & & & & & \\
& Amplitude ratios: & & 0.70 & 1.00 & 0.94 & 0.89 & 0.76 & 0.74 & 0.67 \\
& Errors: & & 0.07 & 0.08 & 0.08 & 0.08 & 0.07 & 0.07 & 0.07 \\
\hline$f_{3}$ & 2.52428 & 0.39615 & 0.0075 & 0.0110 & 0.0104 & 0.0103 & 0.0078 & 0.0077 & 0.0069 \\
Errors & 0.00007 & 0.00001 & & & & & & & \\
& Amplitude ratios: & & 0.68 & 1.00 & 0.94 & 0.94 & 0.71 & 0.70 & 0.63 \\
& Errors: & & 0.11 & 0.13 & 0.13 & 0.13 & 0.11 & 0.11 & 0.11 \\
\hline$f_{4}$ & 2.26550 & 0.44140 & 0.0020 & 0.0036 & 0.0034 & 0.0030 & 0.0027 & 0.0024 & 0.0022 \\
Errors & 0.00022 & 0.00004 & & & & & & & \\
& Amplitude ratios: & & 0.58 & 1.00 & 0.96 & 0.86 & 0.76 & 0.67 & 0.61 \\
& Errors: & & 0.32 & 0.41 & 0.39 & 0.38 & 0.36 & 0.33 & 0.34 \\
\hline$f_{5}$ & 2.59928 & 0.38472 & 0.0028 & 0.0044 & 0.0040 & 0.0039 & 0.0027 & 0.0024 & 0.0024 \\
Errors & 0.00020 & 0.00003 & & & & & & & 0.54 \\
& Amplitude ratios: & & 0.64 & 1.00 & 0.90 & 0.90 & 0.61 & 0.55 & 0.54 \\
& Errors: & & 0.27 & 0.33 & 0.31 & 0.32 & 0.27 & 0.26 & 0.27 \\
\hline$f_{6}$ & 2.46060 & 0.40640 & 0.0029 & 0.0039 & 0.0040 & 0.0039 & 0.0029 & 0.0028 & 0.0026 \\
& 0.00019 & 0.00003 & & & & & & & \\
& Amplitude ratios: & & 0.72 & 0.97 & 1.00 & 0.96 & 0.71 & 0.68 & 0.63 \\
& Errors: & & 0.30 & 0.35 & 0.35 & 0.35 & 0.30 & 0.29 & 0.30 \\
\hline & & & & & & & &
\end{tabular}

Table 22. HD 207223.

\begin{tabular}{|c|c|c|c|c|c|c|c|c|c|}
\hline HD 207223 & & & $\bar{U}$ & $\overline{\overline{B 1}}$ & $\overline{\bar{B}}$ & $\overline{\overline{B 2}}$ & $\overline{\overline{V 1}}$ & $\overline{\bar{V}}$ & $\overline{\bar{G}}$ \\
\hline & Mean magnitudes: & & 6.9961 & 6.6026 & 5.6300 & 7.0305 & 6.8966 & 6.1730 & 7.2762 \\
\hline & Errors: & & 0.0008 & 0.0010 & 0.0009 & 0.0010 & 0.0009 & 0.0009 & 0.0010 \\
\hline & Amplitude errors: & & 0.0012 & 0.0014 & 0.0013 & 0.0014 & 0.0013 & 0.0013 & 0.0014 \\
\hline HD 207223 & Frequency $\left(\mathrm{d}^{-1}\right)$ & $\operatorname{Period}(d)$ & $\bar{U}$ & $B 1$ & $B$ & $B 2$ & $V 1$ & $V$ & $G$ \\
\hline \multirow{4}{*}{$\begin{array}{r}f_{1} \\
\text { Errors }\end{array}$} & 0.38542 & 2.59457 & 0.0072 & 0.0108 & 0.0095 & 0.0095 & 0.0074 & 0.0074 & 0.0066 \\
\hline & 0.00010 & 0.00065 & & & & & & & \\
\hline & Amplitude ratios: & & 0.66 & 1.00 & 0.88 & 0.87 & 0.68 & 0.68 & 0.61 \\
\hline & Errors: & & 0.14 & 0.19 & 0.17 & 0.17 & 0.15 & 0.15 & 0.15 \\
\hline
\end{tabular}

Table 23. HD 211699.

\begin{tabular}{|c|c|c|c|c|c|c|c|c|c|}
\hline HD 211699 & & & $\overline{\bar{U}}$ & $\overline{\overline{B 1}}$ & $\overline{\bar{B}}$ & $\overline{\overline{B 2}}$ & $\overline{\overline{V 1}}$ & $\overline{\bar{V}}$ & $\overline{\bar{G}}$ \\
\hline & Mean magnitudes: & & 9.9568 & 9.5352 & 8.5691 & 9.9679 & 9.8425 & 9.1226 & 10.2255 \\
\hline & Errors: & & 0.0012 & 0.0016 & 0.0014 & 0.0014 & 0.0012 & 0.0011 & 0.0012 \\
\hline & Amplitude errors: & & 0.0017 & 0.0022 & 0.0020 & 0.0020 & 0.0017 & 0.0016 & 0.0017 \\
\hline HD 211699 & Frequency $\left(\mathrm{d}^{-1}\right)$ & Period $(d)$ & $\bar{U}$ & $B 1$ & $B$ & $B 2$ & $V 1$ & $V$ & $G$ \\
\hline & 0.93280 & 1.07204 & 0.0307 & 0.0451 & 0.0423 & 0.0413 & 0.0321 & 0.0311 & 0.0284 \\
\hline \multirow[t]{3}{*}{ Errors } & 0.00003 & 0.00003 & & & & & & & \\
\hline & Amplitude ratios: & & 0.68 & 1.00 & 0.94 & 0.92 & 0.71 & 0.69 & 0.63 \\
\hline & Errors: & & 0.05 & 0.07 & 0.06 & 0.06 & 0.05 & 0.05 & 0.05 \\
\hline \multirow{4}{*}{$\begin{array}{r}f_{2} \\
\text { Errors }\end{array}$} & 1.12637 & 0.88781 & 0.0043 & 0.0094 & 0.0095 & 0.0094 & 0.0046 & 0.0070 & 0.0073 \\
\hline & 0.00019 & 0.00015 & & & & & & & \\
\hline & Amplitude ratios: & & 0.46 & 0.99 & 1.00 & 0.99 & 0.49 & 0.73 & 0.77 \\
\hline & Errors: & & 0.21 & 0.32 & 0.30 & 0.30 & 0.21 & 0.23 & 0.24 \\
\hline \multirow{4}{*}{$\begin{array}{r}f_{3} \\
\text { Errors }\end{array}$} & 1.16331 & 0.85962 & 0.0110 & 0.0145 & 0.0141 & 0.0129 & 0.0116 & 0.0111 & 0.0104 \\
\hline & 0.00008 & 0.00006 & & & & & & & \\
\hline & Amplitude ratios: & & 0.76 & 1.00 & 0.97 & 0.89 & 0.80 & 0.77 & 0.72 \\
\hline & Errors: & & 0.17 & 0.22 & 0.20 & 0.20 & 0.17 & 0.16 & 0.16 \\
\hline \multirow{4}{*}{$\begin{array}{r}f_{4} \\
\text { Errors }\end{array}$} & 0.19363 & 5.16449 & 0.0126 & 0.0175 & 0.0165 & 0.0155 & 0.0129 & 0.0123 & 0.0118 \\
\hline & 0.00007 & 0.00187 & & & & & & & \\
\hline & Amplitude ratios: & & 0.72 & 1.00 & 0.95 & 0.89 & 0.74 & 0.70 & 0.68 \\
\hline & Errors: & & 0.13 & 0.18 & 0.17 & 0.16 & 0.13 & 0.13 & 0.13 \\
\hline
\end{tabular}


Table 24. HD 218396.

\begin{tabular}{|c|c|c|c|c|c|c|c|c|c|}
\hline HD 218396 & & & $\overline{\bar{U}}$ & $\overline{B B 1}$ & $\overline{B B}$ & $\overline{B 2}$ & $\overline{V 1}$ & $\bar{V}$ & $\overline{\bar{G}}$ \\
\hline & Mean magnitudes: & & 6.6524 & 6.2587 & 5.3186 & 6.7531 & 6.6774 & 5.9601 & 7.0853 \\
\hline & Errors: & & 0.0008 & 0.0011 & 0.0011 & 0.0011 & 0.0009 & 0.0008 & 0.0008 \\
\hline & Amplitude errors: & & 0.0012 & 0.0015 & 0.0015 & 0.0015 & 0.0013 & 0.0012 & 0.0012 \\
\hline HD 218396 & Frequency $\left(\mathrm{d}^{-1}\right)$ & $\operatorname{Period}(d)$ & $U$ & $B 1$ & $B$ & $B 2$ & $V 1$ & $V$ & $G$ \\
\hline \multirow{4}{*}{$\begin{array}{r}J 1 \\
\text { Errors }\end{array}$} & 1.98054 & 0.50491 & 0.0137 & 0.0221 & 0.0220 & 0.0222 & 0.0175 & 0.0161 & 0.0154 \\
\hline & 0.00004 & 0.00001 & & & & & & & \\
\hline & Amplitude ratios: & & 0.62 & 0.99 & 0.99 & 1.00 & 0.79 & 0.73 & 0.69 \\
\hline & Errors: & & 0.07 & 0.10 & 0.09 & 0.10 & 0.08 & 0.07 & 0.07 \\
\hline \multirow{4}{*}{$\begin{array}{r}f_{2} \\
\text { Errors }\end{array}$} & 1.73261 & 0.57716 & 0.0134 & 0.0210 & 0.0204 & 0.0205 & 0.0166 & 0.0158 & 0.0144 \\
\hline & 0.00004 & 0.00001 & & & & & & & \\
\hline & Amplitude ratios: & & 0.64 & 1.00 & 0.97 & 0.98 & 0.79 & 0.75 & 0.68 \\
\hline & Errors: & & 0.07 & 0.10 & 0.10 & 0.10 & 0.08 & 0.08 & 0.07 \\
\hline \multirow{4}{*}{$\begin{array}{r}f_{3} \\
\text { Errors }\end{array}$} & 0.76762 & 1.30273 & 0.0075 & 0.0121 & 0.0116 & 0.0116 & 0.0099 & 0.0093 & 0.0083 \\
\hline & 0.00007 & 0.00013 & & & & & & & \\
\hline & Amplitude ratios: & & 0.62 & 1.00 & 0.95 & 0.96 & 0.81 & 0.77 & 0.68 \\
\hline & Errors: & & 0.12 & 0.18 & 0.17 & 0.17 & 0.15 & 0.14 & 0.13 \\
\hline
\end{tabular}

Table 25. HD 221866.

\begin{tabular}{|c|c|c|c|c|c|c|c|c|c|}
\hline HD 221866 & & & $\overline{\bar{U}}$ & $\overline{B 1}$ & $\overline{\bar{B}}$ & $\overline{\overline{B 2}}$ & $\overline{\overline{V 1}}$ & $\overline{\bar{V}}$ & $\overline{\bar{G}}$ \\
\hline & Mean magnitudes: & & 8.2668 & 7.7669 & 6.8081 & 8.2177 & 8.1670 & 7.4528 & 8.5775 \\
\hline & Errors: & & 0.0008 & 0.0010 & 0.0009 & 0.0009 & 0.0008 & 0.0008 & 0.0008 \\
\hline & Amplitude errors: & & 0.0011 & 0.0014 & 0.0013 & 0.0013 & 0.0011 & 0.0011 & 0.0011 \\
\hline HD 221866 & Frequency $\left(\mathrm{d}^{-1}\right)$ & $\operatorname{Period}(d)$ & $\bar{U}$ & $B 1$ & $B$ & $B 2$ & $V 1$ & $\bar{V}$ & $G$ \\
\hline & 0.83843 & 1.19271 & 0.0193 & 0.0257 & 0.0247 & 0.0228 & 0.0184 & 0.0186 & 0.0176 \\
\hline Errors & 0.00003 & 0.00004 & & & & & & & \\
\hline & Amplitude ratios: & & 0.75 & 1.00 & 0.96 & 0.88 & 0.71 & 0.72 & 0.69 \\
\hline & Errors: & & 0.06 & 0.08 & 0.07 & 0.07 & 0.06 & 0.06 & 0.06 \\
\hline & 0.87722 & 1.13996 & 0.0201 & 0.0276 & 0.0260 & 0.0237 & 0.0191 & 0.0195 & 0.0174 \\
\hline Errors & 0.00003 & 0.00004 & & & & & & & \\
\hline & Amplitude ratios: & & 0.73 & 1.00 & 0.94 & 0.86 & 0.69 & 0.71 & 0.63 \\
\hline & Errors: & & 0.06 & 0.07 & 0.07 & 0.07 & 0.05 & 0.05 & 0.05 \\
\hline & 1.71562 & 0.58288 & 0.0069 & 0.0090 & 0.0084 & 0.0091 & 0.0071 & 0.0069 & 0.0074 \\
\hline Errors & 0.00008 & 0.00003 & & & & & & & \\
\hline & Amplitude ratios: & & 0.76 & 0.99 & 0.93 & 1.00 & 0.79 & 0.76 & 0.81 \\
\hline & Errors: & & 0.16 & 0.21 & 0.20 & 0.21 & 0.17 & 0.16 & 0.17 \\
\hline
\end{tabular}

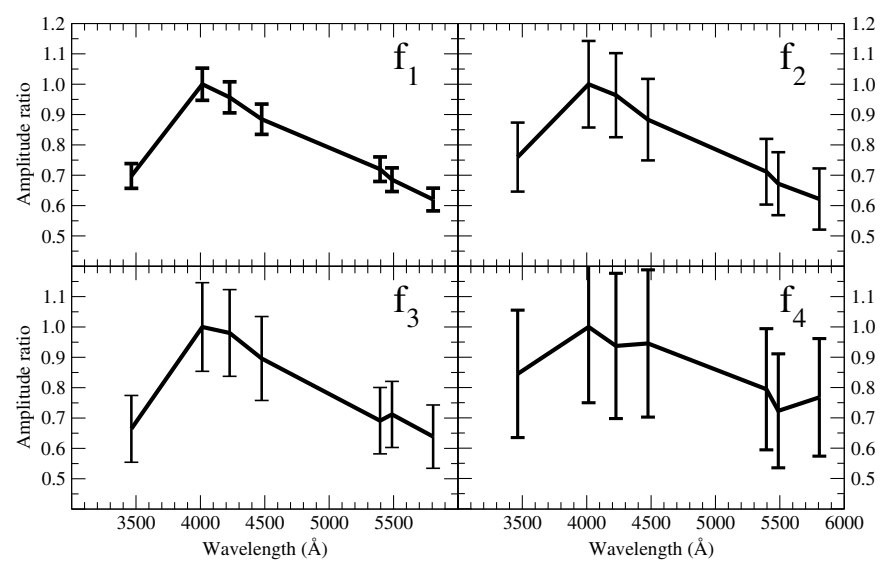

Fig. 2. The ratios of the amplitudes in the Geneva colours (with reference to the highest amplitude) of the four frequencies found for the star HD 277

g-mode pulsations in an extended sample of $\gamma$ Dor stars so far, and it constitutes a useful reference for future interpretations of the oscillatory behaviour of such pulsators.
Acknowledgements. This research was made possible thanks to the financial support from the Fund for Scientific Research - Flanders (FWO), under projects G.0178.02 and G.0332.06. The Leuven authors additionally are supported by the Research Council of K.U. Leuven under grant GOA/2008/04. The research leading to these results has received funding from the European Research Council under the European Community's Seventh Framework Programme (FP7/2007-2013)/ERC grant agreement No. 227224 (PROSPERITY). We are indebted to our colleagues of the Geneva Observatory who reduced the MERCATOR data. This research has made use of the NASA's Astrophysics Data System and the SIMBAD astronomical database operated at the CDS in Strasbourg, France.

\section{References}

Aerts, C., \& Kaye, A. B. 2001, ApJ, 553, 814

Aerts, C., Eyer, L., \& Kestens, E. 1998, A\&A, 337, 790

Aerts, C., Cuypers, J., De Cat, P., et al. 2004, A\&A, 415, 1079

Balona, L. A., \& Stobie, R. S. 1979, MNRAS, 189, 649

Breger, M., Handler, G., Garrido, R., et al. 1997, A\&A, 324, 566

Breger, M., Handler, G., Garrido, R., et al. 1999, A\&A, 349, 225

Cuypers, J., \& Minarini, R. 1998, in A Half Century of Stellar Pulsation Interpretation, ed. P. A. Bradley, \& J. A. Guzik, ASP Conf. Ser., 135, 218

Cuypers, J., Goossens, K., Schoenaers, C., De Cat, P., \& Aerts, C. 2006, Commun. Asteroseismol., 147, 52

De Cat, P., Eyer, L., Cuypers, J., et al. 2006, A\&A, 449, 281

De Cat, P., Briquet, M., Aerts, C., et al. 2007, A\&A, 463, 243 
De Ridder, J., Cuypers, J., De Cat, P., et al. 2004, in IAU Colloq., 193, Variable Stars in the Local Group, ed. D. W. Kurtz, \& K. R. Pollard, ASP Conf. Ser., 310,263

Dupret, M.-A., Grigahcène, A., Garrido, R., et al. 2005, MNRAS, 360, 1143

Fekel, F. C., Warner, P. B., \& Kaye, A. B. 2003, AJ, 125, 2196

Golay, M. 1966, in Spectral Classification and Multicolour Photometry, ed. K. Loden, L. O. Loden, \& U. Sinnerstad, IAU Symp., 24, 262

Griffin, R. F. 2006, The Observatory, 126, 119

Guinan, E. F., Bochanski, J. J., Depasquale, J. M., Ribas, I., \& McCook, G. P. 2001, Information Bulletin on Variable Stars, 5062, 1

Guzik, J. A., Kaye, A. B., Bradley, P. A., Cox, A. N., \& Neuforge, C. 2000, ApJ, 542, L57

Handler, G. 1999, MNRAS, 309, L19

Henry, G. W., \& Fekel, F. C. 2002, PASP, 114, 988

Henry, G. W., \& Fekel, F. C. 2003, AJ, 126, 3058

Henry, G. W., Fekel, F. C., Kaye, A. B., \& Kaul, A. 2001, AJ, 122, 3383

Henry, G. W., Fekel, F. C., \& Henry, S. M. 2005, AJ, 129, 2815

Henry, G. W., Fekel, F. C., \& Henry, S. M. 2007, AJ, 133, 1421

Jankov, S., Mathias, P., Chapellier, E., Le Contel, J.-M., \& Sareyan, J.-P. 2006,

A\&A, 453, 1041
Kaye, A. B. 1998, Information Bulletin on Variable Stars, 4596, 1

Kaye, A. B., Handler, G., Krisciunas, K., Poretti, E., \& Zerbi, F. M. 1999, PASP,

$$
111,840
$$

Kaye, A. B., Gray, R. O., \& Griffin, R. F. 2004, PASP, 116, 558

Koen, C., \& Eyer, L. 2002, MNRAS, 331, 45

Kunzli, M., North, P., Kurucz, R. L., \& Nicolet, B. 1997, A\&AS, 122, 51

Lomb, N. R. 1976, Ap\&SS, 39, 447

Martín, S., Bossi, M., \& Zerbi, F. M. 2003, A\&A, 401, 1077

Mathias, P., Le Contel, J.-M., Chapellier, E., et al. 2004, A\&A, 417, 189

Michel, E., Baglin, A., Auvergne, M., et al. 2008, Science, 322, 558

Montgomery, M. H., \& O’Donoghue, D. 1999, Delta Scuti Star Newsletter, 13, 28

Rufener, F. 1989, A\&AS, 78, 469

Rufener, F., \& Nicolet, B. 1988, A\&A, 206, 357

Scargle, J. D. 1982, ApJ, 263, 835

Schoenaers, C., \& Cuypers, J. 2004, in IAU Colloq., 193, Variable Stars in the Local Group, ed. D. W. Kurtz, \& K. R. Pollard, ASP Conf. Ser., 310, 283

Stellingwerf, R. F. 1978, ApJ, 224, 953

Watson, R. D. 1988, Ap\&SS, 140, 255

Zerbi, F. M., Rodríguez, E., Garrido, R., et al. 1999, MNRAS, 303, 275 